\title{
LIPSCHITZ CONDITIONS, TRIANGULAR RATIO METRIC, AND QUASICONFORMAL MAPPINGS
}

\author{
Jiaolong Chen, Parisa Hariri, Riku Klén and Matti Vuorinen \\ Hunan Normal University, Department of Mathematics \\ Changsha, P. R. China; jiaolongchen@sina.com \\ University of Turku, Department of Mathematics and Statistics \\ 20014 Turku, Finland; parisa.hariri@utu.fi \\ Massey University, Auckland, New Zealand, and \\ University of Turku, Department of Mathematics and Statistics \\ 20014 Turku, Finland; riku.klen@utu.fi \\ University of Turku, Department of Mathematics and Statistics \\ 20014 Turku, Finland; vuorinen@utu.fi
}

\begin{abstract}
The triangular ratio metric is studied in subdomains of the complex plane and Euclidean $n$-space. Various inequalities are proven for this metric. The main results deal with the behavior of this metric under quasiconformal maps. We also study the smoothness of metric disks with small radii.
\end{abstract}

\section{Introduction}

A significant part of geometric function theory deals with the behavior of distances under well known classes of mappings such as Möbius transformations, bilipschitz maps or quasiconformal mappings. Thus measurement of distances in terms of metrics is a common tool in function theory and frequently hyperbolic metrics or metrics of hyperbolic type are used in addition to Euclidean or chordal distance. Many authors have contributed to this development in recent years. See for instance [H, HIMPS, KL, PT]. A survey of these developments is given in [Vu2].

The triangular ratio metric is defined as follows for a domain $G \subsetneq \mathbf{R}^{n}$ and $x, y \in G$ :

$$
s_{G}(x, y)=\sup _{z \in \partial G} \frac{|x-y|}{|x-z|+|z-y|} \in[0,1] .
$$

Clearly, the supremum in the definition (1.1) of $s_{G}$ is attained at some point $z \in \partial G$, but finding this point is a nontrivial problem even for the case when $G$ is the unit disk. Hästö [H, Theorem 6.1] proved that $s_{G}$ satisfies the triangle inequality and developed theory for metrics more general than $s_{G}$ and generalized the work of Barrlund [BA]. Very recently, the geometry of the balls of $s_{G}$ for some special domains was studied in [HKLV]. Our goal here is to continue the study of this metric and to explore its behavior under Möbius transformations, quasiconformal and quasiregular mappings. We also give upper and lower bounds for this metric in terms of other metrics in

doi:10.5186/aasfm.2015.4039

2010 Mathematics Subject Classification: Primary 51M10, 30C65. theorem.

Key words: Quasiconformal maps, quasiregular maps, conformal invariance, distortion 
several domains such as the unit ball, the upper half plane and $\mathbf{R}^{n} \backslash\{0\}$, the whole space $\mathbf{R}^{n}$ punctured at the origin. Also some ideas for further work are pointed out.

The paper is divided into sections as follows. In Section 2 we give algorithms for numerically finding the value of $s_{G}(x, y)$, for instance, in the case of a domain bounded by a polygon. In Section 3 we develop the main ideas of this paper and relate the triangular ratio metric to other well-known metrics of geometric function theory such as the hyperbolic metric of the unit ball or half-space or to the distance ratio metric of a domain $G \subset \mathbf{R}^{n}$. In Section 5 we apply these results and well-known distortion results of quasiconformal maps to study how the triangular ratio metric behaves under quasiconformal and quasiregular mappings. In Section 4 we study the smoothness of the boundaries of $s$-disks in a triangle and in a rectangle. We now proceed to formulate some of our main results.

Theorem 1.2. (1) Let $f: \mathbf{H}^{n} \rightarrow \mathbf{H}^{n}$ be a $K$-quasiregular mapping. Then for $x, y \in \mathbf{H}^{n}$ we have

$$
s_{\mathbf{H}^{n}}(f(x), f(y)) \leq \lambda_{n}^{1-\alpha}\left(s_{\mathbf{H}^{n}}(x, y)\right)^{\alpha}, \quad \alpha=K^{1 /(1-n)},
$$

where $\lambda_{n} \in\left[4,2 e^{n-1}\right), \lambda_{2}=4$, is the Grötzsch ring constant depending only on $n$ [Vu1, Lemma 7.22].

(2) Let $f: \mathbf{B}^{n} \rightarrow \mathbf{B}^{n}$ be a $K$-quasiregular mapping. Then for $x, y \in \mathbf{B}^{n}$ we have

$$
s_{\mathbf{B}^{n}}(f(x), f(y)) \leq 2^{\alpha} \lambda_{n}^{1-\alpha}\left(s_{\mathbf{B}^{n}}(x, y)\right)^{\alpha}, \quad \alpha=K^{1 /(1-n)} .
$$

(3) Let $f: \mathbf{B}^{n} \rightarrow \mathbf{H}^{n}$ be a $K$-quasiregular mapping. Then for $x, y \in \mathbf{B}^{n}$ we have

$$
s_{\mathbf{H}^{n}}(f(x), f(y)) \leq 2^{\alpha} \lambda_{n}^{1-\alpha}\left(s_{\mathbf{B}^{n}}(x, y)\right)^{\alpha}, \quad \alpha=K^{1 /(1-n)} .
$$

(4) Let $f: \mathbf{H}^{n} \rightarrow \mathbf{B}^{n}$ be a $K$-quasiregular mapping. Then for $x, y \in \mathbf{H}^{n}$ we have

$$
s_{\mathbf{B}^{n}}(f(x), f(y)) \leq \lambda_{n}^{1-\alpha}\left(s_{\mathbf{H}^{n}}(x, y)\right)^{\alpha}, \quad \alpha=K^{1 /(1-n)} .
$$

Theorem 1.3. Let $G=\mathbf{R}^{n} \backslash\{0\}$, and $f: G \rightarrow G$ be a $K$-quasiconformal mapping with $f(\infty)=\infty$, and let $z, w$ be two distinct points in $G$ and $\alpha=K^{1 /(1-n)}$. Then

$$
s_{f G}(f(z), f(w)) \leq \frac{1}{P_{5}(n, K)}\left(s_{G}(z, w)\right)^{\alpha}, \quad s_{G}(z, w)=\frac{|z-w|}{|z|+|w|},
$$

where $P_{5}(n, K) \rightarrow 1, K \rightarrow 1$, and $P_{5}(n, K)$ is defined in Lemma 5.8.

Of particular interest is the special case $K=1$ of Theorems 1.2 and 1.3. Clearly, Theorem 1.3 is sharp in this case and the same is true about Theorem 1.2 (1). The question about the best constant in Theorem 1.2 (2) deserves some attention for the case when $K=1=\alpha$. The constant on the right hand side is then 2 .

For a detailed study of this constant we define for $a \in(0,1)$ the class $C(a)$ of all Möbius transformations $h: \mathbf{B}^{n} \rightarrow \mathbf{B}^{n}$ with $|h(0)|=a$ and the constant

$$
L(a)=\sup \left\{s_{\mathbf{B}^{n}}(h(x), h(y)) / s_{\mathbf{B}^{n}}(x, y): x, y \in \mathbf{B}^{n}, x \neq y, h \in C(a)\right\} .
$$

Theorem 1.5. For $n=2, L(a) \geq 1+a$.

Theorem 1.5 shows that for $K=1$ the constant 2 in Theorem 1.2 (2) cannot be replaced by a smaller constant (independent of $a$ ).

Conjecture 1.6. Our numerical experiments for $n=2$ suggest that $L(a)=1+a$. 
In Theorem 3.31 we show that $L(a) \leq \frac{1+a}{1-a}$.

For a domain $G \subset \mathbf{R}^{n}, x, y \in G$, we define the $j$-metric by

$$
j_{G}(x, y)=\log \left(1+\frac{|x-y|}{\min \left\{d_{G}(x), d_{G}(y)\right\}}\right),
$$

where $d_{G}(z)=d(z, \partial G)$. We will omit the subscript $G$ if it is clear from context. This metric has found numerous applications in geometric function theory, see [HIMPS, Vu1]. We also define

$$
p_{G}(x, y)=\frac{|x-y|}{\sqrt{|x-y|^{2}+4 d_{G}(x) d_{G}(y)}} .
$$

We next formulate some of our comparison results between metrics.

Theorem 1.7. Let $G$ be a proper subdomain of $\mathbf{R}^{n}$. Then for all $x, y \in G$ we have

$$
p_{G}(x, y) \leq \frac{1}{\sqrt{2}} j_{G}(x, y), \quad \text { and } \quad s_{G}(x, y) \leq \frac{1}{\log 3} j_{G}(x, y),
$$

where the constant $\frac{1}{\log 3} \approx 0.91$ is the best possible.

Theorem 1.8. (1) Let $t \in(0,1)$ and $m \in\{j, p, s\}$. There exists a constant $c_{m}=c_{m}(t)>1$ such that for all $x, y \in \mathbf{B}^{n}$ with $|x|,|y|<t$ we have

$$
m_{\mathbf{B}^{n}}(x, y) \leq c_{m} m_{\mathbf{R}^{n} \backslash\left\{e_{1}\right\}}(x, y) .
$$

Moreover, $c_{m}(t) \rightarrow 1$ as $t \rightarrow 0$ and $c_{m}(t) \rightarrow \infty$ as $t \rightarrow 1$, for all $m \in\{j, p, s\}$.

(2) Let $G \subset \mathbf{R}^{n}, x \in G, t \in(0,1)$ and $m \in\{j, p, s\}$. Then there exists a constant $c_{m}=c_{m}(t)$ such that for all $y, z \in G \backslash \mathbf{B}^{n}\left(x, t d_{G}(x)\right)$ we have

$$
m_{G \backslash\{x\}}(y, z) \leq c_{m} m_{G}(y, z) .
$$

Moreover, the constant is best possible as $t \rightarrow 1$. This means that $c_{j}, c_{p}, c_{s} \rightarrow$ 2 as $t \rightarrow 1$.

We also study the geometry of balls of the $s$-metric. We use the notation

$$
B_{s_{G}}(x, r)=\left\{z \in G: s_{G}(x, z)<r\right\}
$$

for the balls of the $s$-metric. First we show, for $n=2$, that disks of small enough radii have smooth boundaries and our main result here is Theorem 1.9.

Let us denote $T_{\frac{\pi}{6}, 2}$ the equilateral triangle with vertices $(0,0),(\sqrt{3}, 1),(\sqrt{3},-1)$, and $R_{a, b}$ the rectangle with vertices $(a, b),(a,-b),(-a, b),(-a,-b)$, where $a \geq b>0$.

Theorem 1.9. (1) Let $G=T_{\frac{\pi}{6}, 2}, x=\left(x_{1}, x_{2}\right) \in G, r>0$. Then the metric ball $B_{s_{G}}(x, r)$ is smooth if and only if $r \leq r_{0}$ or $r \leq r_{1}$, where

$$
r_{0}=\min \left\{\frac{2\left|x_{2}\right|}{|x|}, \frac{\left|x_{2}\right|-\sqrt{3} x_{1}+2}{\sqrt{\left(x_{1}-\sqrt{3}\right)^{2}+\left(1-\left|x_{2}\right|\right)^{2}}}\right\}, \quad r_{1}=\frac{\sqrt{3} x_{1}-2-\left|x_{2}\right|}{\sqrt{\left(x_{1}-\sqrt{3}\right)^{2}+\left(1-\left|x_{2}\right|\right)^{2}}} .
$$

(2) Let $G=R_{a, b}, x=\left(x_{1}, x_{2}\right) \in G, r>0$. Then the metric ball $B_{s_{G}}(x, r)$ is smooth if and only if $r \leq r_{2}$ or $r \leq r_{3}$, where

$$
r_{2}=\min \left\{\frac{\left|x_{2}\right|}{b}, \frac{\left(a-\left|x_{1}\right|\right)-\left(b-\left|x_{2}\right|\right)}{\sqrt{\left(a-\left|x_{1}\right|\right)^{2}+\left(b-\left|x_{2}\right|\right)^{2}}}\right\},
$$


and

$$
r_{3}=\min \left\{\frac{\left|x_{1}\right|}{a}, \frac{\left(b-\left|x_{2}\right|\right)-\left(a-\left|x_{1}\right|\right)}{\sqrt{\left(a-\left|x_{1}\right|\right)^{2}+\left(b-\left|x_{2}\right|\right)^{2}}}\right\} .
$$

\section{Algorithms for numerical computation of $s_{G}$}

The hyperbolic metric $\rho_{\mathbf{H}^{n}}$ and $\rho_{\mathbf{B}^{n}}$ of the upper half plane $\mathbf{H}^{n}=\left\{\left(x_{1}, \ldots, x_{n}\right) \in\right.$ $\left.\mathbf{R}^{n}: x_{n}>0\right\}$ and of the unit ball $\mathbf{B}^{n}=\left\{z \in \mathbf{R}^{n}:|z|<1\right\}$ can be defined as weighted metrics with the weight functions $w_{\mathbf{H}^{n}}(x)=1 / x_{n}$ and $w_{\mathbf{B}^{n}}(x)=2 /\left(1-|x|^{2}\right)$, respectively. This definition as such is rather abstract and for applications concrete formulas are needed. By [B, p. 35] we have

$$
\cosh \rho_{\mathbf{H}^{n}}(x, y)=1+\frac{|x-y|^{2}}{2 x_{n} y_{n}}
$$

for all $x, y \in \mathbf{H}^{n}$, and by [B, p. 40] we have

$$
\sinh \frac{\rho_{\mathbf{B}^{n}}(x, y)}{2}=\frac{|x-y|}{\sqrt{1-|x|^{2}} \sqrt{1-|y|^{2}}}
$$

and

$$
\text { th } \frac{\rho_{\mathbf{B}^{n}}(x, y)}{2}=\frac{|x-y|}{\sqrt{|x-y|^{2}+\left(1-|x|^{2}\right)\left(1-|y|^{2}\right)}}=\frac{|x-y|}{|x|\left|x^{*}-y\right|}, \quad x^{*}=\frac{x}{|x|^{2}},
$$

for all $x, y \in \mathbf{B}^{n} \backslash\{0\}$. As shown in [HKLV, Theorem 4.2] we have

$$
s_{\mathbf{H}^{n}}(x, y)=\operatorname{th} \frac{\rho_{\mathbf{H}^{n}}(x, y)}{2}=\frac{|x-y|}{|x-\bar{y}|},
$$

for all $x, y \in \mathbf{H}^{n}$, where $\bar{y}$ is the reflection of $y$ with respect to $\partial \mathbf{H}^{n}$. See also (2.8) below. Unfortunately, there is no formula similar to (2.4) for the case of $s_{\mathbf{B}^{n}}$. Therefore inequalities for $s_{\mathbf{B}^{n}}$ are needed, see Section 3 below.

Explicit formulas for $s_{G}(x, y)$ are known only for a few particular cases. Our goal is to list several domains for which we have written algorithms in the MATLAB language. The definition of $s_{G}(x, y)$ readily shows that the supremum is attained and that a point $z \in \partial G$ with $s_{G}(x, y)=\frac{|x-y|}{|x-z|+|z-y|}$ is located on the maximal ellipse with foci $x$ and $y$ and contained in $\bar{G}$. The point $z$ is called an extremal point. Finding this maximal ellipse is however a difficult task even for $\mathbf{B}^{2}$. In the course of this research we have extensively made use of experiments using the algorithms in this section. In particular, Conjecture 1.6 is based on these algorithms.

Algorithm 2.5. $s_{\mathbf{B}^{2}}$. Let $x, y \in \mathbf{B}^{2}$ and $z \in \partial \mathbf{B}^{2}$ be such that

$$
s_{\mathbf{B}^{2}}(x, y)=\frac{|x-y|}{|x-z|+|z-y|} .
$$

The point $z$ can be found by choosing $m$ equally spaced points on the smaller arc on $\partial \mathbf{B}^{2}$ between $\frac{x}{|x|}$ and $\frac{y}{|y|}$ and selecting the point $z$ that minimizes the expression $|x-z|+|z-y|$ among the chosen points, say for $m=1000$.

Algorithm 2.7. $s_{\mathbf{H}^{2}}$. Suppose that $x, y \in \mathbf{H}^{2}$ are two distinct points. An extremal point $z \in \partial \mathbf{H}^{2}=\mathbf{R}$ for $s_{\mathbf{H}^{2}}(x, y)$ minimizes the sum

$$
|x-z|+|z-y|=|x-z|+|z-\bar{y}|,
$$


where $\bar{y}$ is as in formula (2.4). Therefore $z$ is the unique point of intersection of the segment $[x, \bar{y}]$ with the real axis. In conclusion,

$$
s_{\mathbf{H}^{2}}(x, y)=\frac{|x-y|}{|x-\bar{y}|} .
$$

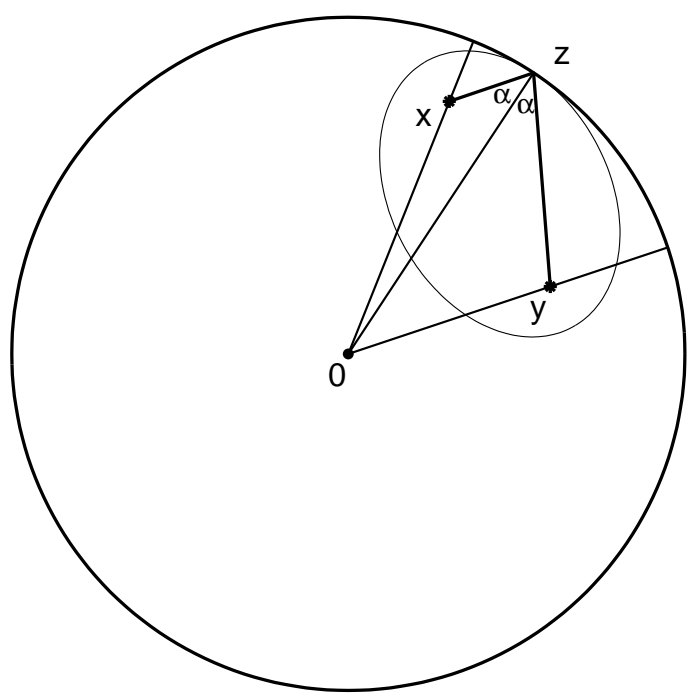

Figure 1. The maximal ellipse with foci $x$ and $y$ and contained in $\overline{\mathbf{B}^{2}}$.

Remark 2.9. Sometimes it is convenient to write the formula (2.8) in a different form which we give now. Suppose that $x, y \in \mathbf{H}^{2}$ with $d(x)=d\left(x, \partial \mathbf{H}^{2}\right) \geq d(y)$. Let $w$ be a point on the segment $[x, \bar{x}] \cap \mathbf{H}^{2}$ with $d(w)=d(y)$ and $\alpha=\measuredangle(w-y, x-y)$. Then clearly $|w-y|=|\bar{w}-\bar{y}|=|x-y| \cos \alpha$ and $\sin \alpha=(d(x)-d(y)) /|x-y|$. This yields

$$
|y-w|=\sqrt{|x-y|^{2}-(d(x)-d(y))^{2}}
$$

and also, by the Pythagorean Theorem,

$$
|x-\bar{y}|^{2}=|x-\bar{w}|^{2}+|\bar{w}-\bar{y}|^{2}=|x-y|^{2}+4 d(x) d(y) .
$$

In conclusion, the formula (2.8) can also be written as

$$
s_{\mathbf{H}^{2}}(x, y)=\frac{|x-y|}{\sqrt{|x-y|^{2}+4 d(x) d(y)}} .
$$

Therefore we see by (2.4) that

$$
p_{\mathbf{H}^{2}}(x, y)=s_{\mathbf{H}^{2}}(x, y)=\operatorname{th} \frac{\rho_{\mathbf{H}^{2}}(x, y)}{2} .
$$

Algorithm 2.10. $s_{R}, R$ is a rectangle. Given distinct $x, y$ in a rectangle $R$, the extremal boundary point $z$ as in (1.1) must be located on one of the four sides $T_{j}, j=1, \ldots, 4$, of $R$. If $y_{j}$ is the reflection point of $y$ with respect to side $T_{j}, j=$ $1, \ldots, 4$, then $z_{j}=\left[x, y_{j}\right] \cap \partial R$ and

$$
s_{R}(x, y)=\frac{|x-y|}{\min \left\{\left|x-y_{j}\right|: j=1,2,3,4\right\}} .
$$

Algorithm 2.12. $s_{A}, A$ is a sector. Let $\alpha \in(0, \pi)$ and $A=\{z \in \mathbf{C}: 0<\arg z<$ $\alpha\}$. Given $x, y \in A$, the extremal point $z \in \partial A$ for $s_{A}(x, y)$ has only two options: it 
is located either on the real axis $\{x \in \mathbf{R}: x \geq 0\}$ or on the ray $\{t \exp i \alpha: t>0\}$. In the first case by (2.8)

$$
s_{A}(x, y)=\frac{|x-y|}{|x-\bar{y}|}
$$

whereas in the second case again by $(2.8)$

$$
s_{A}(x, y)=\frac{|x-y|}{\left|x-y_{2}\right|},
$$

where $y_{2}=|y| \exp i(2 \alpha-\arg y)$. In conclusion, in both cases

$$
s_{A}(x, y)=\frac{|x-y|}{\min \left\{|x-\bar{y}|,\left|x-y_{2}\right|\right\}} .
$$

This idea can be extended in a straightforward way to triangles and other convex polygons.

Algorithm 2.14. $s_{P}, P$ is a polygon. Suppose that $v_{1}, v_{2}, \ldots, v_{m}$ are points in the plane such that the polygon with these points as vertices is a bounded Jordan domain. The method is based on exhaustive tabulation of function values and choosing the optimal point on $\partial P$. We parameterize $\partial P$ using the polygonal curve length as a parameter, measured from $v_{1}$ via the points $v_{j}$. Then this real parameter varies on $[0, L]$ where

$$
L=\sum_{j=1}^{m}\left|v_{j}-v_{j+1}\right|,
$$

and we agree that $v_{m+1}=v_{1}$. The parametrization $z:[0, L] \longrightarrow \partial P$ enables us to find all the competing points for the definition of $s_{P}(x, y)$. Then finding $s_{P}(x, y)$ becomes a 1-dimensional minimization problem, which can be solved by exhaustive tabulation.

\section{Comparison results for $s_{G}$}

The goal of this section is to find inequalities between distances of points in terms of simple expressions. Problems of two kinds are considered. First, if $m_{G}$ is a metric defined in a domain $G \subset \mathbf{R}^{n}, x, y \in G$, then we compare $m_{G}(x, y)$ and $m_{G_{1}}(x, y)$ where $G_{1}$ is a simple domain. Second, if we have two metrics $e_{G}$ and $d_{G}$ on a domain $G \subset \mathbf{R}^{n}$, then we estimate $e_{G}(x, y)$ in terms of $d_{G}(x, y)$. In several cases, this comparison is carried out not in the whole domain but in $B^{n}\left(x_{0}, \lambda d\left(x_{0}, \partial G\right)\right)$ where $x_{0} \in G$ is a fixed point and $\lambda \in(0,1)$ is a constant. In some results we consider the case of $\mathbf{B}^{n}$. Some examples of the metrics we use are the hyperbolic and distance ratio metrics and the $s$ and $v$ metrics.

From the definition (1.1) of $s_{G}$ it is clear that $s_{G}$ has three important properties:

(a) monotonicity with respect to domain, i.e., if $D_{1}, D_{2} \subset \mathbf{R}^{n}$ are domains with $D_{1} \subset D_{2}$ and $x, y \in D_{1}$, then $s_{D_{1}}(x, y) \geq s_{D_{2}}(x, y)$.

(b) Sensitivity to boundary variation, i.e., if $D \subset \mathbf{R}^{n}$ is a domain and $x_{0} \in D$, then the numerical values of $s_{D}(x, y)$ and $s_{D \backslash\left\{x_{0}\right\}}(x, y)$ are not comparable if $x, y$ are very close to $x_{0}$.

(c) For fixed $x, y \in G$, one extremal boundary point $z \in \partial G$ determines the numerical value of $s_{G}(x, y)$. 
Our goal is to find various inequalities for $s_{G}$ in terms of expressions that are explicit. In particular, we hope to get rid of the supremum in (1.1), and hope to use expressions that have the above properties (a)-(c). Most of these expressions define metrics and we will show that these metrics are locally quantitatively equivalent.

For a domain $G \subset \mathbf{R}^{n}, x, y \in G$, we define the visual angle metric [KLVW] by

$$
v_{G}(x, y)=\sup \{\measuredangle(x, z, y): z \in \partial G\} .
$$

The metrics $j_{G}, v_{G}$ and $s_{G}$ have the aforementioned three properties (a)-(c) and $p_{G} \leq 1, v_{G} \leq \pi$ while $j_{G}$ is unbounded. All of the expressions $s_{G}, v_{G}, j_{G}, p_{G}$ are invariant under similarity transformations.

Remark 3.1. Because the inequality $p_{\mathbf{B}^{2}}(t, 0)+p_{\mathbf{B}^{2}}(0,-t)>p_{\mathbf{B}^{2}}(t,-t)$, fails for small $t$, we see that $p_{G}$ is not a metric.

Lemma 3.2. [Vu1, Lemma 2.41(2)], [AVV, Lemma 7.56] Let $G \in\left\{\mathbf{B}^{n}, \mathbf{H}^{n}\right\}$, and let $\rho_{G}$ stand for the respective hyperbolic metric. Then for all $x, y \in G$

$$
j_{G}(x, y) \leq \rho_{G}(x, y) \leq 2 j_{G}(x, y) .
$$

The following theorem solves a question posed in [HKLV, Open problem 3.2].

Theorem 3.3. Let $G$ be a proper subdomain of $\mathbf{R}^{n}$. Then for all $x, y \in G$ we have

$$
s_{G}(x, y) \leq \frac{1}{\log 3} j_{G}(x, y)
$$

and the constant $\frac{1}{\log 3} \approx 0.91$ is the best possible.

Proof. Let us fix the points $x$ and $y$. By rescaling the domain we may assume that $|x-y|=1$. We can also assume that $d(x) \leq d(y)$, because otherwise we can swap the points.

We denote $t=d(x)>0$. Now

$$
j_{G}(x, y)=\log \left(1+\frac{1}{t}\right)
$$

and we divide the proof into two cases: $t \leq \frac{1}{2}$ and $t>\frac{1}{2}$.

We assume first that $t \leq \frac{1}{2}$. Now $j_{G}(x, y) \geq \log 3$ and since $s_{G}(x, y) \leq 1$ we have

$$
s_{G}(x, y) \leq 1 \leq \frac{j_{G}(x, y)}{\log 3} .
$$

We assume then that $t>\frac{1}{2}$. We want to maximize $s_{G}(x, y)$ in terms of $t$. Now

$$
s_{G}(x, y) \leq s_{\mathbf{B}^{n}(x, t) \cup \mathbf{B}^{n}(y, t)}(x, y)=\frac{|x-y|}{|x-z|+|y-z|}=\frac{1}{2 t},
$$

$z \in \partial\left(\mathbf{B}^{n}(x, t) \cup \mathbf{B}^{n}(y, t)\right)$, and we want to find a lower bound for the function

$$
f(t)=\frac{j_{G}(x, y)}{s_{G}(x, t)} \geq 2 t \log \left(1+\frac{1}{t}\right), \quad t>\frac{1}{2} .
$$

We can show that $g(t)=\frac{\log (1+t)}{t}$ is decreasing for $t$, because

$$
g^{\prime}(t)=\frac{\frac{t}{1+t}-\log (1+t)}{t^{2}} \leq \frac{\frac{t}{1+t}-\frac{2 t}{2+t}}{t^{2}} \leq 0,
$$


so it is increasing for $\frac{1}{t}$, thus $f(t)$ is increasing. We collect $f(t)>f\left(\frac{1}{2}\right)=\log 3$ and the claimed inequality is proved.

The constant $\frac{1}{\log 3}$ can be easily verified to be the best possible by investigating the domain $G=\mathbf{R}^{n} \backslash\{0\}$. For any $x \in G$ selecting $y=-x$ gives $s_{G}(x, y)=1$ and $j_{G}(x, y)=\log 3$.

Lemma 3.4. (1) If $x, y \in G \subset \mathbf{R}^{n}$ and $G$ is convex, then

$$
s_{G}(x, y) \leq p_{G}(x, y) .
$$

Here equality holds for all $x, y \in G$ if $G=\mathbf{H}^{n}$.

(2) For $x, y \in G \subset \mathbf{R}^{n}$,

$$
p_{G}(x, y) \leq \sqrt{2} s_{G}(x, y) .
$$

Proof. (1) Suppose that $z \in \partial G$ is an extremal boundary point for the $s$-metric for which the equality holds in (1.1). We draw a line $L$ through $z$, tangent to $\partial G$. Let $\bar{y}$ be the reflection of $y$ in the line $L$. By geometry, see Remark 2.9,

$$
|x-z|+|z-y|=|x-\bar{y}|=\sqrt{|x-y|^{2}+4 d_{1}(x) d_{1}(y)},
$$

$d_{1}(x)=d(x, L), d_{1}(y)=d(y, L)$. Because $G$ is convex it is clear that $L$ is outside $G$, but $d(x), d(y)$ are the shortest distances from $x, y$ to $\partial G$, so obviously $d(x) \leq d_{1}(x)$, $d(y) \leq d_{1}(y)$, thus

$$
\begin{aligned}
s_{G}(x, y) & =\frac{|x-y|}{|x-z|+|z-y|}=\frac{|x-y|}{\sqrt{|x-y|^{2}+4 d_{1}(x) d_{1}(y)}} \\
& \leq \frac{|x-y|}{\sqrt{|x-y|^{2}+4 d(x) d(y)}}=p_{G}(x, y) .
\end{aligned}
$$

(2) Fix $x, y \in G, z \in \partial G$, such that $d(x)=|x-z|$. By symmetry we may assume $d(x) \leq d(y)$ and then

$$
s_{G}(x, y) \geq \frac{|x-y|}{|x-y|+2 d(x)} .
$$

Now by $[A V V, 1.58(13)]$ and (3.5)

$$
\begin{aligned}
p_{G}(x, y) & \leq \frac{|x-y|}{\sqrt{|x-y|^{2}+4 d(x)^{2}}} \leq \frac{|x-y|}{2^{1 / 2-1}(|x-y|+2 d(x))} \\
& \leq \frac{\sqrt{2}|x-y|}{|x-y|+2 d(x)} \leq \sqrt{2} s_{G}(x, y) .
\end{aligned}
$$

It is easy to see that convexity cannot be omitted from Lemma 3.4 (1). For instance if $G=\mathbf{R}^{2} \backslash\{0\}$ and $x=(0,1)=-y$, then the inequality in Lemma 3.4 (1) fails.

Lemma 3.6. For $x, y \in \mathbf{B}^{n}$ we have

$$
s_{\mathbf{B}^{n}}(x, y) \geq s_{\mathbf{B}^{n}}\left(x_{s}, y_{s}\right)=\frac{|x-y|}{\sqrt{|x-y|^{2}+4(1-|m|)^{2}}},
$$


where $m=\frac{x_{0}+y_{0}}{2}$ and $x_{0}, y_{0} \in \partial \mathbf{B}^{n}$ are the points of intersection of the line through $x$ and $y$ with $\partial \mathbf{B}^{n},|x-y|=\left|x_{s}-y_{s}\right|,\left|x_{s}\right|=\left|y_{s}\right|$ and further

$$
\begin{aligned}
|m| & =\frac{\sqrt{|x|^{2}|y|^{2}-(x \cdot y)^{2}}}{|x-y|}, \\
x_{s} & =x_{0}+\frac{y_{0}-x_{0}}{\left|y_{0}-x_{0}\right|}\left(\sqrt{1-|m|^{2}}-\frac{|x-y|}{2}\right), \\
y_{s} & =y_{0}+\frac{x_{0}-y_{0}}{\left|x_{0}-y_{0}\right|}\left(\sqrt{1-|m|^{2}}-\frac{|x-y|}{2}\right),
\end{aligned}
$$

and hence

$$
s_{\mathbf{B}^{n}}(x, y) \geq \frac{|x-y|^{2}}{|x-y|^{4}+4\left(|x-y|-\sqrt{|x|^{2}|y|^{2}-(x \cdot y)^{2}}\right)^{2}} .
$$

Proof. If we move $x, y \in \mathbf{B}^{n}$ to $x_{s}, y_{s} \in \mathbf{B}^{n}$ which are symmetric with respect to midpoint $m$ of the segment $\left[x_{0}, y_{0}\right]$, then we see easily that the extremal ellipse with foci $x_{s}, y_{s}$ is larger than the extremal ellipse with foci $x, y$ and hence by (1.1),

$$
s_{\mathbf{B}^{n}}(x, y) \geq s_{\mathbf{B}^{n}}\left(x_{s}, y_{s}\right)=\frac{|x-y|}{\sqrt{|x-y|^{2}+4(1-|m|)^{2}}} .
$$

Here $|m|$ is the shortest distance from the origin to the line $\overline{x y}$, which by the Law of Cosines, $|m|=\frac{\sqrt{|x|^{2}|y|^{2}-(x \cdot y)^{2}}}{|x-y|}$, and therefore

$$
s_{\mathbf{B}^{n}}\left(x_{s}, y_{s}\right)=\frac{|x-y|^{2}}{|x-y|^{4}+4\left(|x-y|-\sqrt{|x|^{2}|y|^{2}-(x \cdot y)^{2}}\right)^{2}},
$$

and the proof is complete.

Lemma 3.7. For $x, y \in \mathbf{B}^{n}$ with $|x|>|y|, y_{r}=x-\frac{x}{|x|}|x-y|=-\frac{x}{|x|}(|x|-|x-y|)$,

$$
s_{\mathbf{B}^{n}}(x, y) \geq s_{\mathbf{B}^{n}}\left(x, y_{r}\right)=\frac{|x-y|}{|x-y|+2(1-t)} \equiv w(x, y), \quad t=\max \{|x|,|y|\} .
$$

Proof. Note that $y_{r} \in[x,-x]$ and $|x-y|=\left|x-y_{r}\right|$. By geometric properties of the ellipse it is clear that $s_{\mathbf{B}^{n}}(x, y) \geq s_{\mathbf{B}^{n}}\left(x, y_{r}\right)$ and thus

$$
\begin{aligned}
s_{\mathbf{B}^{n}}(x, y) & =\sup _{z \in \partial G} \frac{|x-y|}{|x-z|+|z-y|} \geq s_{\mathbf{B}^{n}}\left(x, y_{r}\right) \\
& =\frac{|x-y|}{|x-y|+2(1-t)}, \quad t=\max \{|x|,|y|\} .
\end{aligned}
$$

Lemma 3.8. For all $x, y \in \mathbf{B}^{n}$ we have

$$
p_{\mathbf{B}^{n}}(x, y) \leq \text { th } \frac{\rho_{\mathbf{B}^{n}}(x, y)}{2} \leq 2 p_{\mathbf{B}^{n}}(x, y) .
$$

Proof. The second inequality follows from Lemma 3.4 and Theorem 3.23. For the first inequality clearly

$$
\left(1-|x|^{2}\right)\left(1-|y|^{2}\right)=(1-|x|)(1-|y|)(1+|x|)(1+|y|) \leq 4(1-|x|)(1-|y|),
$$


so

$$
\begin{aligned}
\operatorname{th} \frac{\rho_{\mathbf{B}^{n}}(x, y)}{2} & =\frac{|x-y|}{\sqrt{|x-y|^{2}+\left(1-|x|^{2}\right)\left(1-|y|^{2}\right)}} \\
& \geq \frac{|x-y|}{\sqrt{|x-y|^{2}+4 d(x) d(y)}}=p_{\mathbf{B}^{n}}(x, y) .
\end{aligned}
$$

Theorem 3.10. If $z \in G, 0<\lambda<1, x, y \in \mathbf{B}^{n}(z, \lambda d(z))$, then

$$
\begin{aligned}
& s_{\mathbf{B}^{n}(z, d(z))}(x, y) \leq C j_{\mathbf{B}^{n}(z, d(z))}(x, y), \quad C=\frac{2(1-\lambda)}{1+2 \lambda}, \\
& j_{\mathbf{B}^{n}(z, d(z))}(x, y) \leq \frac{2(1+\lambda)}{1-\lambda} s_{\mathbf{B}^{n}(z, d(z))}(x, y) .
\end{aligned}
$$

Proof. From $x, y \in \mathbf{B}^{n}(z, \lambda d(z))$ it follows that

$$
\frac{|x-y|}{d(z)} \leq 2 \lambda
$$

Because for all $x, y \in \mathbf{B}^{n}(z, \lambda d(z)), w \in \partial G$, the inequality

$$
|x-w|+|y-w| \geq 2(1-\lambda) d(z)
$$

holds, we see that

$$
s_{\mathbf{B}^{n}(z, d(z))}(x, y) \leq \frac{|x-y|}{2(1-\lambda) d(z)},
$$

and by $\log (1+t) \geq \frac{2 t}{2+t}$, for $t \geq 0$, and (3.13) we see that

$$
j_{\mathbf{B}^{n}(z, d(z))}(x, y) \geq \log \left(1+\frac{|x-y|}{(1+\lambda)(d(z))}\right) \geq \frac{\frac{2|x-y|}{(1+\lambda)(d(z))}}{2+\frac{|x-y|}{(1+\lambda)(d(z))}} \geq \frac{|x-y|}{(1+2 \lambda)(d(z))} .
$$

Hence it suffices to choose $C=\frac{2(1-\lambda)}{1+2 \lambda}$. have

For the second part observing that for $w \in \mathbf{B}^{n}(z, \lambda d(z)), d(w) \geq(1-\lambda) d(z)$ we

$$
j_{\mathbf{B}^{n}(z, d(z))}(x, y) \leq \log \left(1+\frac{|x-y|}{(1-\lambda)(d(z))}\right) \leq \frac{|x-y|}{(1-\lambda)(d(z))} .
$$

On the other hand, setting $w=z+d(z) \frac{y-z}{|y-z|}$ we see that

$$
\begin{aligned}
|x-w|+|y-w| & \leq|x-y|+|y-w|+|y-w| \\
& \leq|x-y|+2 d(z) \leq 2(1+\lambda) d(z)
\end{aligned}
$$

and hence

Now it suffices to find $C$ such that

$$
s_{\mathbf{B}^{n}(z, d(z))}(x, y) \geq \frac{|x-y|}{2(1+\lambda) d(z)} .
$$

$$
\frac{|x-y|}{2(1+\lambda) d(z)} \geq C \frac{|x-y|}{(1-\lambda)(d(z))}
$$

so we may choose $C=\frac{2(1+\lambda)}{1-\lambda}$, and the proof is complete.

Theorem 3.14. If $z \in G, 0<\lambda<1, x, y \in \mathbf{B}^{n}(z, \lambda d(z))$, then

$$
j_{G}(x, y) \leq C p_{G}(x, y), \quad C=\frac{2}{1-\lambda} .
$$


Proof. By symmetry we may assume that $d(x) \leq d(y)$. Then by $\log (1+t) \leq$ $t, t>0$ we have

$$
j_{G}(x, y) \leq \frac{|x-y|}{\min \{d(x), d(y)\}}=\frac{|x-y|}{d(x)} .
$$

On the other hand by the assumption we get $d(z) \leq \frac{1}{1-\lambda} \min \{d(x), d(y)\}$, and

$$
\begin{gathered}
\frac{1-\lambda}{1+\lambda} \leq \frac{d(x)}{d(y)} \leq \frac{1+\lambda}{1-\lambda}, \\
p_{G}(x, y)=\frac{|x-y|}{\sqrt{|x-y|^{2}+4 d(x) d(y)}} \geq \frac{|x-y|}{\sqrt{\left(2 \lambda \frac{d(x)}{1-\lambda}\right)^{2}+4 d(x) \frac{1+\lambda}{1-\lambda} d(x)}} \geq \frac{1-\lambda}{2} \cdot \frac{|x-y|}{d(x)} .
\end{gathered}
$$

We see that

$$
j_{G}(x, y) \leq \frac{|x-y|}{d(x)} \leq C \frac{1-\lambda}{2} \cdot \frac{|x-y|}{2} \leq C p_{G}(x, y)
$$

holds if $C \geq \frac{2}{1-\lambda}$, and the proof is complete.

Theorem 3.15. If $x, y \in G \subset \mathbf{R}^{n}$, then

$$
p_{G}(x, y) \leq \frac{1}{\sqrt{2}} j_{G}(x, y) .
$$
Then

Proof. Fix $x, y \in G$. By relabeling the points we may assume that $d(x) \leq d(y)$.

$$
p_{G}(x, y) \leq \frac{|x-y|}{\sqrt{|x-y|^{2}+4 d(x)^{2}}}, \quad \text { and } \quad j_{G}(x, y)=\log \left(1+\frac{|x-y|}{d(x)}\right) .
$$

Write $t=|x-y| / d(x)$ and observe that

$$
j_{G}(x, y)=\log (1+t) \geq \frac{2 t}{2+t}, \quad p_{G}(x, y) \leq \frac{t}{\sqrt{t^{2}+4}}
$$

It is enough to find a constant $C$ such that

$$
\frac{2 t}{2+t} \geq C \frac{t}{\sqrt{t^{2}+4}}
$$

for all $t \geq 0$. Easy calculation shows that we can choose $C=\sqrt{2}$.

Corollary 3.16. If $x, y \in G \subset \mathbf{R}^{n}$, and $G$ is convex, then

$$
s_{G}(x, y) \leq \frac{1}{\sqrt{2}} j_{G}(x, y) .
$$

Proof. It follows from Lemma 3.4 (1) and Theorem 3.15.

Proof of Theorem 1.7. The result follows from Theorems 3.3 and 3.15.

Theorem 3.17. (1) For $x, y \in \mathbf{B}^{2}$ we have

$$
v_{\mathbf{B}^{2}}(x, y) \leq 2 j_{\mathbf{B}^{2}}(x, y) .
$$

(2) If $\lambda \in(0,1)$ and $x, y \in \mathbf{B}^{2}(\lambda)$, then

$$
\frac{3\left(1-\lambda^{2}\right)}{2\left(3+\lambda^{2}\right)} j_{\mathbf{B}^{2}}(x, y) \leq v_{\mathbf{B}^{2}}(x, y) .
$$


Proof. (1) By [KLVW, 3.12] we have $v_{\mathbf{B}^{2}}(x, y) \leq \rho_{\mathbf{B}^{2}}(x, y)$. Now the proof follows by Lemma 3.2.

(2) By Lemma 3.2

$$
\sinh \frac{\rho_{\mathbf{B}^{2}}(x, y)}{2} \leq \sinh j_{\mathbf{B}^{2}}(x, y) \leq \sinh \left(\log \left(1+\frac{2 \lambda}{1-\lambda}\right)\right)=\frac{2 \lambda}{1-\lambda^{2}},
$$

and by $[\mathrm{KLVW}, 3.15] \rho_{\mathbf{B}^{2}}^{*} \leq v_{\mathbf{B}^{2}} \leq 2 \rho_{\mathbf{B}^{2}}^{*}$, where

$$
\rho_{\mathbf{B}^{2}}^{*}(x, y)=\arctan \left(\sinh \frac{\rho_{\mathbf{B}^{2}}(x, y)}{2}\right) .
$$

Next by [DC, 1.8]

$$
\frac{3 t}{1+2 \sqrt{1+t^{2}}}<\arctan t<\frac{2 t}{1+\sqrt{1+t^{2}}}
$$

for $t>0$. We further obtain

$$
\begin{aligned}
\rho_{\mathbf{B}^{2}}^{*}(x, y) & =\arctan \left(\sinh \frac{\rho_{\mathbf{B}^{2}}(x, y)}{2}\right) \geq \frac{3 \sinh \frac{\rho_{\mathrm{B}^{2}}(x, y)}{2}}{1+2 \sqrt{1+\sinh ^{2} \frac{\rho_{\mathrm{B}^{2}}(x, y)}{2}}} \\
& \geq \frac{3 \sinh \frac{j_{\mathrm{B}^{2}}(x, y)}{2}}{1+2 \sqrt{1+\left(\frac{2 \lambda}{1-\lambda^{2}}\right)^{2}}}=\frac{3\left(1-\lambda^{2}\right)}{3+\lambda^{2}} \sinh \frac{j_{\mathrm{B}^{2}}(x, y)}{2} \geq \frac{3\left(1-\lambda^{2}\right)}{2\left(3+\lambda^{2}\right)} j_{\mathrm{B}^{2}}(x, y) .
\end{aligned}
$$

Thus

$$
\frac{3\left(1-\lambda^{2}\right)}{2\left(3+\lambda^{2}\right)} j_{\mathbf{B}^{2}}(x, y) \leq v_{\mathbf{B}^{2}}(x, y) .
$$

Theorem 3.18. If $z \in G, \lambda \in(0,1)$, then for $x, y \in \mathbf{B}^{n}(z, \lambda d(z))$,

$$
s_{G}(x, y) \leq\left(\frac{1+\lambda}{1-\lambda}\right) p_{G}(x, y) .
$$

Proof. By monotonicity of $s$-metric and Lemma 3.4 (1)

$$
s_{G}(x, y) \leq s_{\mathbf{B}^{n}(z, d(z))}(x, y) \leq p_{\mathbf{B}^{n}(z, d(z))}(x, y) \leq \frac{|x-y|}{\sqrt{|x-y|^{2}+4(1-\lambda)^{2} d(z)^{2}}} .
$$

If $x, y \in \mathbf{B}^{n}(z, \lambda d(z))$, we easily see that

$$
(1-\lambda) d(z) \leq d_{G}(x) \leq(1+\lambda) d(z) .
$$

Now if we choose $c=\left(\frac{1+\lambda}{1-\lambda}\right)$, then

$$
\frac{|x-y|}{\sqrt{|x-y|^{2}+4(1-\lambda)^{2} d(z)^{2}}} \leq \frac{c|x-y|}{\sqrt{|x-y|^{2}+4(1+\lambda)^{2} d(z)^{2}}} \leq c p_{G}(x, y) .
$$

Theorem 3.21. Let $0<\lambda<1, x, y \in \mathbf{B}^{2}(\lambda)$. Then

(1) $s_{\mathbf{B}^{2}}(x, y) \leq \frac{4\left(3+\lambda^{2}\right)}{3(1+2 \lambda)(1+\lambda)} v_{\mathbf{B}^{2}}(x, y)$,

(2) $v_{\mathbf{B}^{2}}(x, y) \leq \frac{4(1+\lambda)}{1-\lambda} s_{\mathbf{B}^{2}}(x, y)$. 
Proof. (1) By Theorem 3.17 and (3.11),

$$
s_{\mathbf{B}^{2}}(x, y) \leq \frac{4\left(3+\lambda^{2}\right)}{3(1+2 \lambda)(1+\lambda)} v_{\mathbf{B}^{2}}(x, y) .
$$

(2) By Theorem 3.17 and (3.12),

$$
v_{\mathbf{B}^{2}}(x, y) \leq 2 j_{\mathbf{B}^{2}}(x, y) \leq \frac{4(1+\lambda)}{1-\lambda} s_{\mathbf{B}^{2}}(x, y) .
$$

Theorem 3.22. (1) If $\lambda \in(0,1)$ and $x, y \in \mathbf{B}^{2}(\lambda)$, then

$$
v_{\mathbf{B}^{2}}(x, y) \leq \frac{4(1+\lambda)}{(1-\lambda)} p_{\mathbf{B}^{2}}(x, y) \text {. }
$$

(2) If $x, y \in \mathbf{B}^{2}$ with $v_{\mathbf{B}^{2}}(x, y) \in(0, \pi / 2)$, then

$$
p_{\mathbf{B}^{2}}(x, y) \leq v_{\mathbf{B}^{2}}(x, y),
$$

Proof. (1) By Theorems 3.21 and 3.4,

$$
v_{\mathbf{B}^{2}}(x, y) \leq \frac{4(1+\lambda)}{(1-\lambda)} s_{\mathbf{B}^{2}}(x, y) \leq \frac{4(1+\lambda)}{(1-\lambda)} p_{\mathbf{B}^{2}}(x, y) .
$$

(2) By Lemma 3.8 and [KLVW, 3.15] we have

$$
\rho_{\mathbf{B}^{2}}^{*}(x, y)=\arctan \left(\sinh \frac{\rho_{\mathbf{B}^{2}}(x, y)}{2}\right) \leq v_{\mathbf{B}^{2}}(x, y) .
$$

Then

$$
\rho_{\mathbf{B}^{2}}(x, y) \leq 2 \operatorname{arsinh}\left(\tan \left(v_{\mathbf{B}^{2}}(x, y)\right)\right) .
$$

Then if $v_{\mathbf{B}^{2}}(x, y) \in(0, \pi / 2)$,

$$
\begin{aligned}
p_{\mathbf{B}^{2}}(x, y) & \leq \operatorname{th}\left(\operatorname{arsinh}\left(\tan \left(v_{\mathbf{B}^{2}}(x, y)\right)\right)\right) \\
& =\frac{\tan \left(v_{\mathbf{B}^{2}}(x, y)\right)}{\sqrt{1+\tan ^{2}\left(v_{\mathbf{B}^{2}}(x, y)\right)}}=\sin \left(v_{\mathbf{B}^{2}}(x, y)\right) \leq v_{\mathbf{B}^{2}}(x, y) .
\end{aligned}
$$

Theorem 3.23. For $x, y \in \mathbf{B}^{n}$ we have

$$
\text { th }\left(\frac{\rho_{\mathbf{B}^{n}}(x, y)}{2}\right) \leq 2 s_{\mathbf{B}^{n}}(x, y) .
$$

Proof. Suppose first that one of the points $x$ and $y$ is 0 . Without loss of generality, we may suppose that $y=0$. From the definition of $s_{\mathbf{B}^{n}}$ it follows that for $z=\frac{x}{|x|}$

$$
s_{\mathbf{B}^{n}}(x, 0) \geq \frac{|x-0|}{|x-z|+|z-0|}=\frac{|x|}{2-|x|} .
$$

Because

$$
\operatorname{th}\left(\frac{\rho_{\mathbf{B}^{n}}(x, y)}{2}\right)=|x|,
$$

we easily see that the claim holds if one of the points is 0 . The case when both points are 0 is trivial.

By (2.3) and (1.1) it is enough to show that

$$
I \leq 2|x|\left|x^{*}-y\right|, \quad I=\inf _{z \in \partial \mathbf{B}^{n}}|x-z|+|z-y| .
$$

Assume $|y| \leq|x|$. Denote $|y|=t|x|$ for $t \in[0,1], \gamma \in[0, \pi]$, is angle between $[0, x]$ and $[0, y]$. 
Case A. $\gamma \geq \frac{\pi}{2}$. Now

$$
2|x|\left|x^{*}-y\right| \geq 2|x| \frac{1}{|x|}=2,
$$

Moreover, choose $z_{1}=\frac{x}{|x|}$, then

$$
\begin{aligned}
I & \leq\left|x-z_{1}\right|+\left|z_{1}-y\right| \leq 1-|x|+\sqrt{t^{2}|x|^{2}+1+2 t|x|} \\
& =2-|x|+t|x|=2-(|x|(1-t)) \leq 2 .
\end{aligned}
$$

So by (3.25) and (3.26),

Case B. $\gamma \leq \frac{\pi}{2}$.

$$
I \leq 2|x|\left|x^{*}-y\right|
$$

$$
2|x|\left|x^{*}-y\right|=2|| y\left|x-z_{2}\right|=2|| x\left|y-z_{1}\right|,
$$

where $\left|z_{2}\right|=\frac{y}{|y|}$ and $\left|z_{1}\right|=\frac{x}{|x|}$. Next we choose $z$ in the infimum to be the middle point of $z_{1}$ and $z_{2}$ on the unit sphere. This means that $\measuredangle(x, 0, z)=\measuredangle(z, 0, y)=\gamma / 2$ and $|z|=1$. We know that

$$
I \leq|x-z|+|z-y|
$$

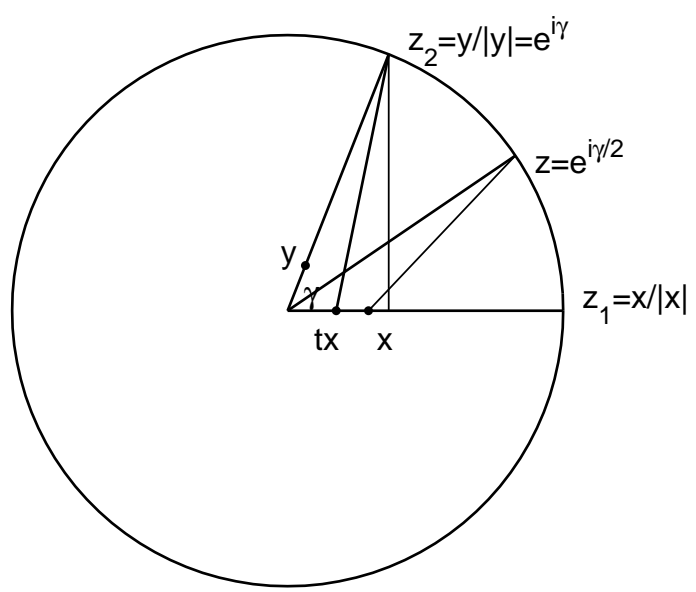

Figure 2. Proof of Theorem 3.23. The case $r=|z-x|>\sin (\gamma)$.

We next show that

$$
p / r \geq 1, \quad p=\left|z_{2}-\right| y|x|, \quad r=|z-x| .
$$

By elementary geometry, applying the properties of the right triangle $\Delta\left(0, z_{2},(\cos \gamma) z_{1}\right)$ and the Law of Cosines, we see that

$$
\begin{aligned}
p & \geq\left|z_{2}-(\cos \gamma) z_{1}\right|=\sin \gamma \geq \sqrt{1+\cos ^{2}(\gamma)-2 \cos (\gamma) \cos (\gamma / 2)} \\
& =\left|z-(\cos \gamma) z_{1}\right| .
\end{aligned}
$$

The second inequality follows because for $\gamma \in(0, \pi / 2)$,

$$
\sin ^{2}(\gamma)>1+\cos ^{2}(\gamma)-2 \cos (\gamma) \cos (\gamma / 2)
$$

by basic trigonometry.

If $r \leq \sin \gamma$, then by (3.29) $p / r \geq 1$ clearly holds. In the remaining case $r=$ $|z-x|>\sin \gamma$. Because $x \in\left[0, z_{1}\right]$, this means by $(3.29)$ that $x \in\left[0,(\cos \gamma) z_{1}\right]$ and hence the angle between the segments $\left[x, z_{2}\right]$ and $[x, 0]$ is more than $\pi / 2$ and hence

$$
p=\left|z_{2}-\right| y|x|>\left|z_{2}-x\right| \text {. }
$$


Finally, we see that $p / r \geq\left|z_{2}-x\right| /|z-x|>1$, because $x$ and $z$ both are in the same half plane determined by the bisecting normal of the segment $\left[z_{2}, z\right]$. Symmetrically we obtain that

$$
|z-y| \leq|| x\left|y-z_{1}\right|
$$

and hence

$$
|x-z|+|z-y| \leq|| y\left|x-z_{2}\right|+|| x\left|y-z_{1}\right|=2|x|\left|x^{*}-y\right|
$$

and the proof is complete.

Corollary 3.30. (1) If $f: \mathbf{H}^{n} \rightarrow \mathbf{H}^{n}$ is a Möbius transformation onto $\mathbf{H}^{n}$, then for all $x, y \in \mathbf{H}^{n}$,

$$
s_{\mathbf{H}^{n}}(f(x), f(y))=s_{\mathbf{H}^{n}}(x, y) .
$$

(2) If $f: \mathbf{H}^{n} \rightarrow \mathbf{B}^{n}$ is a Möbius transformation onto $\mathbf{B}^{n}$, then for all $x, y \in \mathbf{H}^{n}$,

$$
s_{\mathbf{B}^{n}}(f(x), f(y)) \leq s_{\mathbf{H}^{n}}(x, y) .
$$

(3) If $f: \mathbf{B}^{n} \rightarrow \mathbf{H}^{n}$ is a Möbius transformation onto $\mathbf{H}^{n}$, then for all $x, y \in \mathbf{B}^{n}$,

$$
s_{\mathbf{H}^{n}}(f(x), f(y)) \leq 2 s_{\mathbf{B}^{n}}(x, y) .
$$

(4) If $f: \mathbf{B}^{n} \rightarrow \mathbf{B}^{n}$ is a Möbius transformation onto $\mathbf{B}^{n}$, then for all $x, y \in \mathbf{B}^{n}$,

$$
s_{\mathbf{B}^{n}}(f(x), f(y)) \leq 2 s_{\mathbf{B}^{n}}(x, y) .
$$

Proof. It is a basic fact that a Möbius transformation $f: G \rightarrow D=f G$ with $G, D \in\left\{\mathbf{B}^{n}, \mathbf{H}^{n}\right\}$ defines an isometry $f:\left(G, \rho_{G}\right) \rightarrow\left(D, \rho_{D}\right)$ between hyperbolic spaces. This fact combined with (2.4), Lemma 3.8 and Theorem 3.23 yields the proof.

We were led to Conjecture 1.6 by MATLAB experiments. We now show that if the conjecture holds true, then the constant $1+a$ cannot be improved when $n=2$.

Proof of Theorem 1.5. Let $h(z)=\frac{z+a}{1+a z}$. Then $h(0)=a, a>0$. Choose $b$ such that $h(b)=\frac{1+v a}{1+v}, v>0$. Easy calculation yields $b=\frac{1}{1+v(1+a)}$. Since $s_{\mathbf{B}^{2}}(r, t)=\frac{t-r}{2-t-r}$ for $0<r<t$ we see that

$$
\frac{s_{\mathbf{B}^{2}}(h(0), h(b))}{s_{\mathbf{B}^{2}}(0, b)}=\frac{\frac{1+v a}{1+v}-a}{2-a-\frac{1+v a}{1+v}} \cdot \frac{2-\frac{1}{1+v(1+a)}}{\frac{1}{1+v(1+a)}}=\frac{1+2 v(1+a)}{1+2 v} \rightarrow 1+a,
$$

when $v \rightarrow \infty$.

Theorem 3.31. If $f: \mathbf{B}^{n} \rightarrow \mathbf{B}^{n}=f\left(\mathbf{B}^{n}\right)$ is a Möbius transformation with $f(a)=0$, for some $a \in \mathbf{B}^{n}$, then for all distinct points $x, y \in \mathbf{B}^{n}$ we have

$$
\frac{1-|a|}{1+|a|} s_{\mathbf{B}^{n}}(x, y) \leq s_{\mathbf{B}^{n}}(f(x), f(y)) \leq \frac{1+|a|}{1-|a|} s_{\mathbf{B}^{n}}(x, y) .
$$

Proof. If $f(0)=0$ then $f$ is a rotation and there is nothing to prove. Otherwise $f(a)=0$ some $a \neq 0$. Let $f=T_{a}$ be the canonical representation of a Möbius transformation, see [B]. Then with $a^{*}=a /|a|^{2}, r=\sqrt{|a|^{-2}-1}$ we have

$$
\left|T_{a}(x)-T_{a}(y)\right|=\frac{r^{2}|x-y|}{\left|x-a^{*}\right|\left|y-a^{*}\right|} .
$$


If $w \in \partial \mathbf{B}^{n}$, then this formula yields

$$
Q(x, y, w)=\frac{\left|T_{a} x-T_{a} y\right|}{\left|T_{a} x-T_{a} w\right|+\left|T_{a} w-T_{a} y\right|}: \frac{|x-y|}{|x-w|+|w-y|}=\frac{|x-w|+|w-y|}{\beta|x-w|+\gamma|w-y|}
$$

with $\beta=\left|y-a^{*}\right| /\left|w-a^{*}\right|, \gamma=\left|x-a^{*}\right| /\left|w-a^{*}\right|$. Clearly,

$$
\left|w-a^{*}\right| \leq 1+|a|^{-1}, \quad\left|x-a^{*}\right|,\left|y-a^{*}\right| \geq|a|^{-1}-1
$$

and hence

$$
Q(x, y, w) \leq \frac{|x-w|+|w-y|}{|x-w|+|w-y|} \frac{1+|a|}{1-|a|}=\frac{1+|a|}{1-|a|} .
$$

Thus we have for all $x, y \in \mathbf{B}^{n}, w \in \partial \mathbf{B}^{n}$

$$
\frac{\left|T_{a} x-T_{a} y\right|}{\left|T_{a} x-T_{a} w\right|+\left|T_{a} w-T_{a} y\right|} \leq \frac{1+|a|}{1-|a|} \frac{|x-y|}{|x-w|+|w-y|} .
$$

Taking supremum over all $w \in \partial \mathbf{B}^{n}$ yields the second inequality. Because the inverse of a Möbius transformation also is a Möbius transformation, the first inequality follows from the second one.

We compare next $j, p, s$ and $v$ in domains $\mathbf{R}^{n} \backslash\left\{e_{1}\right\}$ and $\mathbf{B}^{n}$. By the monotonicity with respect to domains it is clear that for all $x, y \in \mathbf{B}^{n}$ and $m \in\{j, p, s, v\}$ we have $m_{\mathbf{R}^{n} \backslash\left\{e_{1}\right\}}(x, y) \leq m_{\mathbf{B}^{n}}(x, y)$. Next we consider the comparison in the opposite direction. Let us start by introducing the following lemma.

Lemma 3.32. For $0<b \leq a$ the function

$$
f(x)=\frac{\log (1+a x)}{\log (1+b x)}, \quad x \in(0, \infty),
$$

is decreasing.

Proof. Since

$$
f^{\prime}(x)=\frac{\frac{a}{1+a x} \log (1+b x)-\frac{b}{1+b x} \log (1+a x)}{\log ^{2}(1+b x)},
$$

the inequality $f^{\prime}(x) \leq 0$ is equivalent to

$$
\frac{1+b x}{b} \log (1+b x) \leq \frac{1+a x}{a} \log (1+a x) .
$$

Now we show that the function

$$
g(c)=\frac{1+c x}{c} \log (1+c x)
$$

is increasing on $(0, \infty)$, which implies (3.33) and the assertion. This is clear because $0<b \leq a$ and

$$
g^{\prime}(c)=\frac{c x-\log (1+c x)}{c^{2}}>0
$$

as $\log (1+y)<y$ for $y>0$.

Theorem 3.34. Let $t \in(0,1)$ and $m \in\{j, p, s\}$. There exists a constant $c_{m}=$ $c_{m}(t)>1$ such that for all $x, y \in \mathbf{B}^{n}$ with $|x|,|y|<t$ we have

$$
m_{\mathbf{B}^{n}}(x, y) \leq c_{m} m_{\mathbf{R}^{n} \backslash\left\{e_{1}\right\}}(x, y) .
$$

Moreover, $c_{m}(t) \rightarrow 1$ as $t \rightarrow 0$ and $c_{m}(t) \rightarrow \infty$ as $t \rightarrow 1$, for all $m \in\{j, p, s\}$. 
Proof. We denote $m_{1}=m_{\mathbf{B}^{n}}, m_{2}=m_{\mathbf{R}^{n} \backslash\left\{e_{1}\right\}}$ and find upper bound for $\frac{m_{1}}{m_{2}}$, which gives us $c_{m}$.

Let us start with $m=j$. We denote $z=|x-y| \in[0,2 t)$ and obtain by Lemma 3.32

$$
\begin{aligned}
\frac{j_{1}}{j_{2}} & =\frac{\log \left(1+\frac{z}{\min \{1-|x|, 1-|y|\}}\right)}{\log \left(1+\frac{z}{\min \left\{\left|x-e_{1}\right|,\left|y-e_{1}\right|\right\}}\right)} \leq \frac{\log \left(1+\frac{z}{1-t}\right)}{\log \left(1+\frac{z}{1+t}\right)} \\
& \leq \lim _{z \rightarrow 0} \frac{\log \left(1+\frac{z}{1-t}\right)}{\log \left(1+\frac{z}{1+t}\right)}=\lim _{z \rightarrow 0} \frac{1+t+z}{1-t+z}=\frac{1+t}{1-t}=c_{j}
\end{aligned}
$$

where the second equality follows from l'Hôspital's rule. Obviously $c_{j} \rightarrow 1$ as $t \rightarrow 0$ and $c_{j} \rightarrow \infty$ as $t \rightarrow 1$

Let us now consider $m=p$. Now

$$
\frac{p_{1}^{2}}{p_{2}^{2}}=\frac{|x-y|^{2}+4\left|x-e_{1}\right|\left|y-e_{1}\right|}{|x-y|^{2}+4(1-|x|)(1-|y|)} \leq \frac{4 t^{2}+4(1+t)^{2}}{0+4(1-t)^{2}}=\frac{2 t^{2}+2 t+1}{t^{2}-2 t+1}
$$

and we can choose

$$
c_{p}=\sqrt{\frac{2 t^{2}+2 t+1}{t^{2}-2 t+1}} .
$$

Clearly $c_{p} \rightarrow 1$ as $t \rightarrow 0$ and $c_{p} \rightarrow \infty$ as $t \rightarrow 1$.

Next we set $m=s$ and obtain by geometry

$$
\frac{s_{1}}{s_{2}}=\frac{\left|x-e_{1}\right|+\left|y-e_{1}\right|}{\inf _{z \in \partial \mathbf{B}^{n}}|x-z|+|z-y|} \leq \frac{2(1+t)}{2(1-t)}=\frac{1+t}{1-t}=c_{s} .
$$

Again it is clear that $c_{s} \rightarrow 1$ as $t \rightarrow 0$ and $c_{s} \rightarrow \infty$ as $t \rightarrow 1$.

Note that for the visual angle metric $v$ the result of Theorem 3.34 does not hold. We would need an upper bound for

$$
\frac{v_{\mathbf{B}^{n}}(x, y)}{v_{\mathbf{R}^{n} \backslash\left\{e_{1}\right\}}(x, y)}=\frac{\sup _{z \in \partial \mathbf{B}^{n}} \measuredangle(x, z, y)}{\measuredangle\left(x, e_{1}, y\right)},
$$

but choosing $x$ and $y$ to be distinct points on the $x_{1}$-axis

$$
\sup _{z \in \partial \mathbf{B}^{n}} \measuredangle(x, z, y)>0
$$

and $\measuredangle\left(x, e_{1}, y\right)=0$.

Next result demonstrates the sensitivity to boundary variation. We consider domains $G \subset \mathbf{R}^{n}$ and $G^{\prime}=G \backslash\{x\}$, where $x \in G$. Again by the monotonicity we have $m_{G}(y, z) \leq m_{G^{\prime}}(y, z)$ for all $y, z \in G^{\prime}$ and $m \in\{j, p, s, v\}$.

Theorem 3.35. Let $G \subset \mathbf{R}^{n}, x \in G, t \in(0,1)$ and $m \in\{j, p, s\}$. Then there exists a constant $c_{m}=c_{m}(t)$ such that for all $y, z \in G \backslash \mathbf{B}^{n}\left(x, t d_{G}(x)\right)$ we have

$$
m_{G \backslash\{x\}}(y, z) \leq c_{m} m_{G}(y, z) .
$$

Moreover, the constant is best possible as $t \rightarrow 1$. This means that $c_{j}, c_{p}, c_{s} \rightarrow 2$ as $t \rightarrow 1$.

Proof. We denote $G^{\prime}=G \backslash\{x\}$ and will find an upper bound for $\frac{m_{G^{\prime}}(y, z)}{m_{G}(y, z)}$.

We consider first the case $m=j$. If $d_{G}(y)=d_{G^{\prime}}(y)$ and $d_{G}(z)=d_{G^{\prime}}(z)$, then there is nothing to prove as $j_{G^{\prime}}(y, z)=j_{G}(y, z)$ and we can choose $c_{j}=1$. We consider next two cases: $d_{G}(y) \neq d_{G^{\prime}}(y), d_{G}(z)=d_{G^{\prime}}(z)$ and $d_{G}(y) \neq d_{G^{\prime}}(y), d_{G}(z) \neq d_{G^{\prime}}(z)$. 
Let us assume $d_{G}(y) \neq d_{G^{\prime}}(y)$ and $d_{G}(z)=d_{G^{\prime}}(z)$ (or by symmetry we could as well assume $d_{G}(y)=d_{G^{\prime}}(y)$ and $\left.d_{G}(z) \neq d_{G^{\prime}}(z)\right)$. Now

$$
\frac{j_{G^{\prime}}(y, z)}{j_{G}(y, z)}=\frac{\log \left(1+\frac{|y-z|}{\min \left\{d_{G^{\prime}}(y), d_{G^{\prime}}(z)\right\}}\right)}{\log \left(1+\frac{|y-z|}{\min \left\{d_{G}(y), d_{G}(z)\right\}}\right)}=\frac{\log \left(1+\frac{|y-z|}{\min \left\{|y-x|, d_{G}(z)\right\}}\right)}{\log \left(1+\frac{|y-z|}{\min \left\{d_{G}(y), d_{G}(z)\right\}}\right)} .
$$

Let us assume that $d_{G}(z) \leq d_{G}(y)$. If $d_{G}(z) \leq|y-x|$ then $j_{G^{\prime}}(y, z)=j_{G}(y, z)$ and there is nothing to prove. If $d_{G}(z) \geq|y-x|$ then

$$
\begin{aligned}
\frac{j_{G^{\prime}}(y, z)}{j_{G}(y, z)} & =\frac{\log \left(1+\frac{|y-z|}{|y-x|}\right)}{\log \left(1+\frac{|y-z|}{d_{G}(z)}\right)} \leq \frac{\log \left(1+\frac{|y-z|}{t d_{G}(x)}\right)}{\log \left(1+\frac{|y-z|}{d_{G}(z)}\right)} \\
& \leq \frac{\log \left(1+\frac{|y-z|}{t d_{G}(x)}\right)}{\log \left(1+\frac{|y-z|}{d_{G}(y)}\right)} \leq \frac{\log \left(1+\frac{|y-z|}{t d_{G}(x)}\right)}{\log \left(1+\frac{|y-z|}{|y-x|+d_{G}(x)}\right)} .
\end{aligned}
$$

If $|x-y| \leq d_{G}(x)$ then we have by Lemma 3.32

$$
\begin{aligned}
\frac{j_{G^{\prime}}(y, z)}{j_{G}(y, z)} & \leq \frac{\log \left(1+\frac{|y-z|}{t d_{G}(x)}\right)}{\log \left(1+\frac{|y-z|}{2 d_{G}(x)}\right)} \leq \lim _{|y-z| / d_{G}(x) \rightarrow 0} \frac{\log \left(1+\frac{|y-z|}{t d_{G}(x)}\right)}{\log \left(1+\frac{|y-z|}{2 d_{G}(x)}\right)} \\
& \leq \lim _{|y-z| / d_{G}(x) \rightarrow 0} \frac{2+\frac{|y-z|}{d_{G}(x)}}{t+\frac{|y-z|}{d_{G}(x)}}=\frac{2}{t} .
\end{aligned}
$$

If $|x-y| \geq d_{G}(x)$ again by Lemma 3.32

$$
\begin{aligned}
\frac{j_{G^{\prime}}(y, z)}{j_{G}(y, z)} & \leq \frac{\log \left(1+\frac{|y-z|}{|y-x|}\right)}{\log \left(1+\frac{|y-z|}{2|y-x|}\right)} \leq \lim _{|y-z| /|y-x| \rightarrow 0} \frac{\log \left(1+\frac{|y-z|}{|y-x|}\right)}{\log \left(1+\frac{|y-z|}{2|y-x|}\right)} \\
& \leq \lim _{|y-z| /|y-x| \rightarrow 0} \frac{2+\frac{|y-z|}{|y-x|}}{1+\frac{|y-z|}{|y-x|}}=2 .
\end{aligned}
$$

Let us then assume $d_{G}(y) \leq d_{G}(z)$. Now $d_{G}(y) \neq d_{G^{\prime}}(y)$ implies $|y-x|<d_{G}(y)$ and thus

$$
\frac{j_{G^{\prime}}(y, z)}{j_{G}(y, z)}=\frac{\log \left(1+\frac{|y-z|}{|y-x|}\right)}{\log \left(1+\frac{|y-z|}{d_{G}(y)}\right)} \leq \frac{\log \left(1+\frac{|y-z|}{|y-x|}\right)}{\log \left(1+\frac{|y-z|}{|y-x|+d_{G}(x)}\right)} .
$$

If $|x-y| \leq d_{G}(x)$ we have by (3.36) and Lemma 3.32

$$
\begin{aligned}
\frac{j_{G^{\prime}}(y, z)}{j_{G}(y, z)} & \leq \frac{\log \left(1+\frac{|y-z|}{t d_{G}(x)}\right)}{\log \left(1+\frac{|y-z|}{2 d_{G}(x)}\right)} \leq \lim _{|y-z| / d_{G}(x) \rightarrow 0} \frac{\log \left(1+\frac{|y-z|}{t d_{G}(x)}\right)}{\log \left(1+\frac{|y-z|}{2 d_{G}(x)}\right)} \\
& \leq \lim _{|y-z| / d_{G}(x) \rightarrow 0} \frac{2+\frac{|y-z|}{d_{G}(x)}}{t+\frac{|y-z|}{d_{G}(x)}}=\frac{2}{t} .
\end{aligned}
$$


If $d_{G}(x) \leq|x-y|$ we have by (3.36) and Lemma 3.32

$$
\begin{aligned}
\frac{j_{G^{\prime}}(y, z)}{j_{G}(y, z)} & \leq \frac{\log \left(1+\frac{|y-z|}{|y-x|}\right)}{\log \left(1+\frac{|y-z|}{2|y-x|}\right)} \leq \lim _{|y-z| /|y-x| \rightarrow 0} \frac{\log \left(1+\frac{|y-z|}{|y-x|}\right)}{\log \left(1+\frac{|y-z|}{2|y-x|}\right)} \\
& \leq \lim _{|y-z| /|y-x| \rightarrow 0} \frac{2+\frac{|y-z|}{|y-x|}}{1+\frac{|y-z|}{|y-x|}}=2 .
\end{aligned}
$$

Let us then assume $d_{G}(y) \neq d_{G^{\prime}}(y)$ and $d_{G}(z) \neq d_{G^{\prime}}(z)$. Now we may assume by symmetry that $|y-x| \leq|z-x|$ and thus

$$
\frac{j_{G^{\prime}}(y, z)}{j_{G}(y, z)}=\frac{\log \left(1+\frac{|y-z|}{|y-x|}\right)}{\log \left(1+\frac{|y-z|}{\min \left\{d_{G}(y), d_{G}(z)\right\}}\right)} \leq \frac{\log \left(1+\frac{|y-z|}{|y-x|}\right)}{\log \left(1+\frac{|y-z|}{|y-x|+d_{G}(x)}\right)}
$$

and this is exactly the same as (3.36) so we know that

$$
\frac{j_{G^{\prime}}(y, z)}{j_{G}(y, z)} \leq \frac{2}{t}
$$

Putting all this together gives us $c_{j}=\frac{2}{t}$.

Let now $m=p$. If $d_{G}(y)=d_{G^{\prime}}(y)$ and $d_{G}(z)=d_{G^{\prime}}(z)$, then there is nothing to prove as $p_{G^{\prime}}(y, z)=p_{G}(y, z)$ and we can choose $c_{p}=1$. We consider next two cases: $d_{G}(y) \neq d_{G^{\prime}}(y), d_{G}(z)=d_{G^{\prime}}(z)$ and $d_{G}(y) \neq d_{G^{\prime}}(y), d_{G}(z) \neq d_{G^{\prime}}(z)$.

Let us assume $d_{G}(y) \neq d_{G^{\prime}}(y)$ and $d_{G}(z)=d_{G^{\prime}}(z)$ (or by symmetry we could as well assume $d_{G}(y)=d_{G^{\prime}}(y)$ and $\left.d_{G}(z) \neq d_{G^{\prime}}(z)\right)$. Now

$$
\begin{aligned}
\frac{p_{G^{\prime}}^{2}(y, z)}{p_{G}^{2}(y, z)} & =\frac{|y-z|^{2}+4 d_{G}(y) d_{G}(z)}{|y-z|^{2}+4 d_{G^{\prime}}(y) d_{G^{\prime}}(z)}=\frac{|y-z|^{2}+4 d_{G}(y) d_{G}(z)}{|y-z|^{2}+4|y-x| d_{G}(z)} \\
& \leq \frac{|y-z|^{2}+4\left(|x-y|+d_{G}(x)\right) d_{G}(z)}{|y-z|^{2}+4|y-x| d_{G}(z)} \\
& =1+\frac{4 d_{G}(x) d_{G}(z)}{|y-z|^{2}+4|y-x| d_{G}(z)} \leq 1+\frac{4 d_{G}(x) d_{G}(z)}{0+4 t d_{G}(x) d_{G}(z)}=1+\frac{1}{t} .
\end{aligned}
$$

Let us then assume $d_{G}(y) \neq d_{G^{\prime}}(y)$ and $d_{G}(z) \neq d_{G^{\prime}}(z)$. Now

$$
\begin{aligned}
\frac{p_{G^{\prime}}^{2}(y, z)}{p_{G}^{2}(y, z)} & =\frac{|y-z|^{2}+4 d_{G}(y) d_{G}(z)}{|y-z|^{2}+4 d_{G^{\prime}}(y) d_{G^{\prime}}(z)}=\frac{|y-z|^{2}+4 d_{G}(y) d_{G}(z)}{|y-z|^{2}+4|y-x||z-x|} \\
& \leq \frac{|y-z|^{2}+4\left(|x-y|+d_{G}(x)\right)\left(|x-z|+d_{G}(x)\right)}{|y-z|^{2}+4|y-x||z-x|} \\
& =1+\frac{4\left(|x-y| d_{G}(x)+|x-z| d_{G}(x)+d_{G}(x)^{2}\right)}{|y-z|^{2}+4|y-x||z-x|} \\
& \leq 1+\frac{4\left(|x-y| d_{G}(x)+|x-z| d_{G}(x)+d_{G}(x)^{2}\right)}{4|y-x||z-x|} \\
& =1+\frac{|x-y| d_{G}(x)}{|y-x||z-x|}+\frac{|x-z| d_{G}(x)}{|y-x||z-x|}+\frac{d_{G}(x)^{2}}{|y-x||z-x|} \\
& \leq 1+\frac{|x-y| d_{G}(x)}{|y-x| t d_{G}(x)}+\frac{|x-z| d_{G}(x)}{t d_{G}(x)|z-x|}+\frac{d_{G}(x)^{2}}{t d_{G}(x) t d_{G}(x)}
\end{aligned}
$$




$$
=1+\frac{2}{t}+\frac{1}{t^{2}}=1+\frac{2 t+1}{t^{2}} .
$$

Combining the cases we obtain $c_{p}=\frac{t+1}{t}$.

Let us finally consider the case $m=s$. Now

$$
\frac{s_{G^{\prime}}(y, z)}{s_{G}(y, z)}=\frac{\inf _{u \in \partial G}|y-u|+|u-z|}{\inf _{u \in \partial G^{\prime}}|y-u|+|u-z|}
$$

and if the infimum in the denominator is obtained at a point $u \in \partial G$, then there is nothing to prove as $s_{G^{\prime}}(y, z)=s_{G}(y, z)$ and we can choose $c_{s}=1$. If this is not the case, then

$$
\begin{aligned}
\frac{s_{G^{\prime}}(y, z)}{s_{G}(y, z)} & =\frac{\inf _{u \in \partial G}|y-u|+|u-z|}{\inf _{u \in \partial G^{\prime}}|y-u|+|u-z|}=\frac{\inf _{u \in \partial G}|y-u|+|u-z|}{|y-x|+|x-z|} \\
& \leq \frac{|x-y|+d_{G}(x)+|x-z|+d_{G}(x)}{|y-x|+|x-z|}=1+\frac{2 d_{G}(x)}{|y-x|+|x-z|} \\
& \leq 1+\frac{2 d_{G}(x)}{2 t d_{G}(x)}=1+\frac{1}{t}
\end{aligned}
$$

and we can choose $c_{s}=1+\frac{1}{t}$.

We see easily that $c_{j}, c_{p}, c_{s} \rightarrow 2$ as $t \rightarrow 1$. We show next that the constants $c_{j}$, $c_{p}$ and $c_{s}$ are best possible. In all three cases we consider $G=\mathbf{R}^{n} \backslash\{0\}$.

We start with the case $m=j$. Let $a>0$. For points $x=e_{1}, y=(1+t) e_{1}$ and $z=(1+t+a) e_{1}$ we have

$$
\frac{j_{G^{\prime}}(y, z)}{j_{G}(y, z)}=\frac{\log \left(1+\frac{a}{t}\right)}{\log \left(1+\frac{a}{1+t}\right)}
$$

and

$$
\frac{j_{G^{\prime}}(y, z)}{j_{G}(y, z)} \rightarrow \frac{\log (1+a)}{\log \left(1+\frac{a}{2}\right)}
$$

as $t \rightarrow 1$. The asymptotic behavior is clear since

$$
\frac{\log (1+a)}{\log \left(1+\frac{a}{2}\right)} \rightarrow 2
$$

as $a \rightarrow 0$.

We next consider the case $m=p$. Let $a \in(0, t]$. For points $x=e_{1}, y=$ $\left(1+\sqrt{t^{2}-a^{2}}\right) e_{1}+a e_{2}$ and $z=\left(1+\sqrt{t^{2}-a^{2}}\right) e_{1}-a e_{2}$ we have $|y-z|=2 a$ and

$$
\frac{p_{G^{\prime}}^{2}(y, z)}{p_{G}^{2}(y, z)}=\frac{|y-z|^{2}+4 d_{G}(y) d_{G}(z)}{|y-z|^{2}+4 d_{G^{\prime}}(y) d_{G^{\prime}}(z)}=\frac{4 a^{2}+4\left(a^{2}+\left(1+\sqrt{t^{2}-a^{2}}\right)^{2}\right)}{4 a^{2}+4 t^{2}} .
$$

Now

$$
\frac{p_{G^{\prime}}^{2}(y, z)}{p_{G}^{2}(y, z)} \rightarrow \frac{4 a^{2}+4\left(a^{2}+\left(1+\sqrt{1-a^{2}}\right)^{2}\right)}{4 a^{2}+4}=\frac{4 a^{2}+8+8 \sqrt{1-a^{2}}}{4 a^{2}+4}
$$

as $t \rightarrow 1$ and

$$
\frac{4 a^{2}+8+8 \sqrt{1-a^{2}}}{4 a^{2}+4} \rightarrow 4
$$

as $a \rightarrow 0$. 
We finally consider the case $m=s$. Let $a \in(0, t]$. For points $x=e_{1}, y=$ $\left(1+\sqrt{t^{2}-a^{2}}\right) e_{1}+a e_{2}$ and $z=\left(1+\sqrt{t^{2}-a^{2}}\right) e_{1}-a e_{2}$ we have $|y-z|=2 a$ and

$$
\begin{aligned}
& \frac{s_{G^{\prime}}(y, z)}{s_{G}(y, z)}=\frac{\frac{2 a}{2 t}}{\frac{2 a}{2 \sqrt{a^{2}+\left(1+\sqrt{t^{2}-a^{2}}\right)^{2}}}}=\frac{\sqrt{a^{2}+\left(1+\sqrt{t^{2}-a^{2}}\right)^{2}}}{t} \\
& \rightarrow \sqrt{a^{2}+\left(1+\sqrt{1-a^{2}}\right)^{2}}
\end{aligned}
$$

as $t \rightarrow 1$ and

$$
\sqrt{a^{2}+\left(1+\sqrt{1-a^{2}}\right)^{2}}=\sqrt{2+2 \sqrt{1-a^{2}}} \rightarrow 2
$$

as $a \rightarrow 0$.

We show next that Theorem 3.35 does not work for the visual angle metric $v$. Let $G=\mathbf{R}^{n} \backslash\{0\}$ and $x=e_{1}$. Now for $y=\frac{e_{1}}{2}$ and $z=2 e_{1}$ we have $v_{G}(y, z)=0$ an $v_{G \backslash\{x\}}(y, z)=\pi$.

Proof of Theorem 1.8. The assertion follows from Theorems 3.34 and 3.35.

\section{Smoothness of $s$-disks with small radii}

In this section, we will consider the smoothness of triangular ratio metric balls in equilateral triangles and rectangles in $\mathbf{R}^{2}$. Let $T_{\frac{\pi}{6}, 2}$ denote the equilateral triangle with vertices $(0,0),(\sqrt{3}, 1),(\sqrt{3},-1)$, and $R_{a, b}$ denote the rectangle with vertices $(a, b),(a,-b),(-a, b),(-a,-b)$, where $a \geq b>0$.

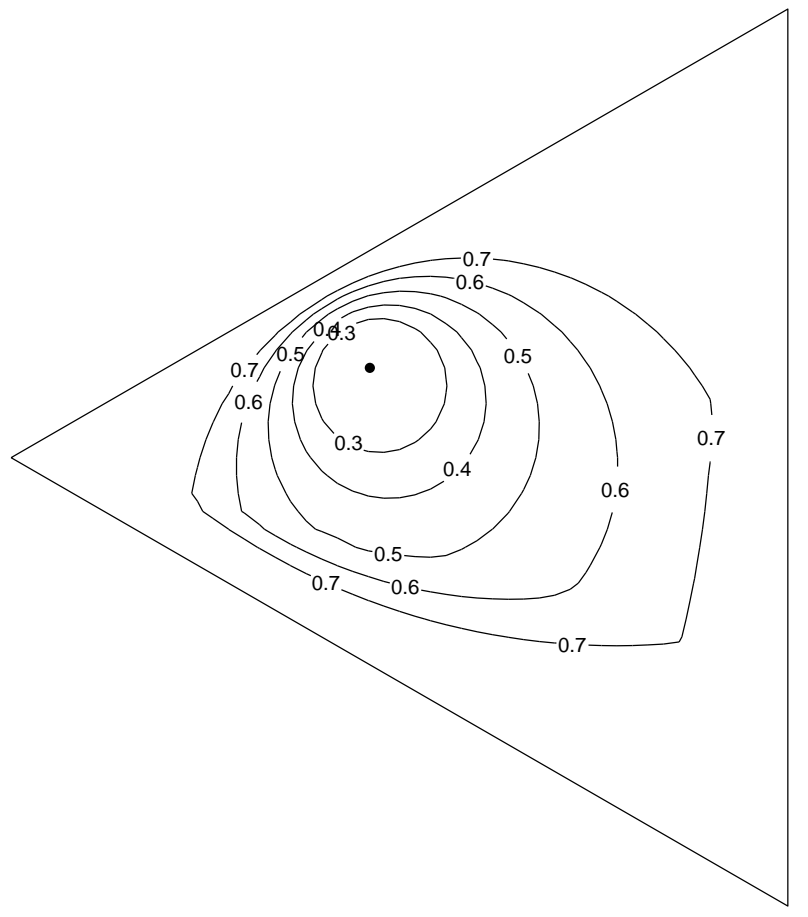

Figure 3. Triangular ratio metric balls $B_{s_{G}}(x, r)$ in $T_{\frac{\pi}{6}, 2}$. 


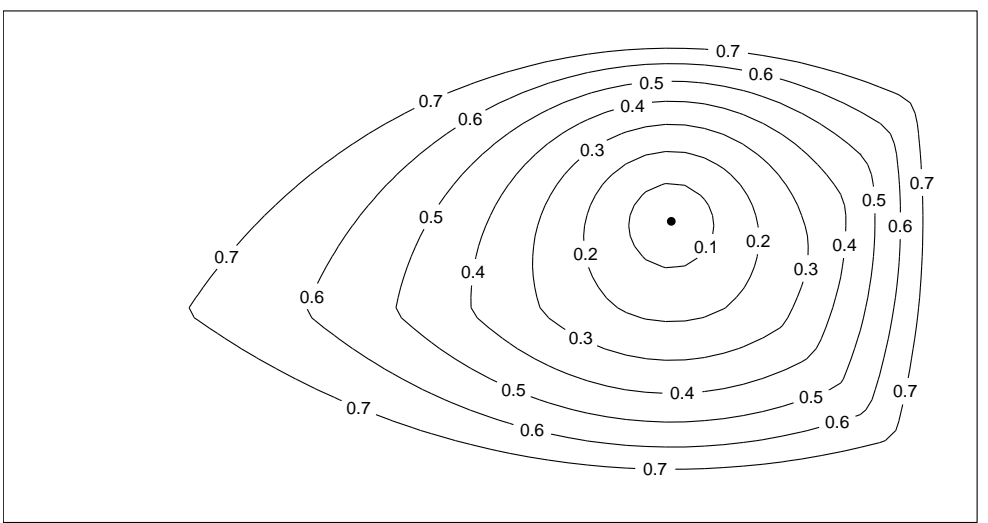

Figure 4. Triangular ratio metric balls $B_{s_{G}}(x, r)$ in $R_{a, b}$.

Lemma 4.1. Let $P \subset \mathbf{R}^{2}$ be a polygon with inner angles less than or equal to $\pi$ and suppose that there are half planes $H_{1}, H_{2}, \ldots, H_{n}$ such that

$$
P=\bigcap_{i=1}^{n} H_{i}
$$

Then for $x \in P$ and $r>0$ we have

$$
B_{s_{P}}(x, r)=\bigcap_{i=1}^{n} B_{s_{H_{i}}}(x, r) .
$$

Proof. Follows from [HKLV, Lemma 5.4].

Proof of Theorem 1.9 Denote by the lines $l_{1}: y_{2}=\frac{\sqrt{3}}{3} y_{1}, l_{2}: y_{2}=-\frac{\sqrt{3}}{3} y_{1}$, and $l_{3}: y_{1}=\sqrt{3}$. For any point $x \in G=T_{\frac{\pi}{6}, 2}$ and $r \in(0,1)$, by Lemma 4.1 , we have

$$
B_{s_{G}}(x, r)=\bigcap_{i=1}^{3} B_{i},
$$

where $B_{i}$ is the corresponding triangular ratio metric ball $B_{s_{G_{i}}}(x, r)$, and $G_{i}$ is the half plane with boundary line $l_{i}$. By elementary computation, we have that

$$
\begin{aligned}
B_{1}:\{y: & \left(y_{1}-\frac{\left(2-r^{2}\right) x_{1}-\sqrt{3} r^{2} x_{2}}{2\left(1-r^{2}\right)}\right)^{2}+\left(y_{2}-\frac{\left(2+r^{2}\right) x_{2}-\sqrt{3} r^{2} x_{1}}{2\left(1-r^{2}\right)}\right)^{2} \\
& \left.<\frac{r^{2}\left(x_{1}-\sqrt{3} x_{2}\right)^{2}}{\left(1-r^{2}\right)^{2}}\right\}, \\
B_{2}:\{y: & \left(y_{1}-\frac{\left(2-r^{2}\right) x_{1}+\sqrt{3} r^{2} x_{2}}{2\left(1-r^{2}\right)}\right)^{2}+\left(y_{2}-\frac{\left(2+r^{2}\right) x_{2}+\sqrt{3} r^{2} x_{1}}{2\left(1-r^{2}\right)}\right)^{2} \\
& \left.<\frac{r^{2}\left(x_{1}+\sqrt{3} x_{2}\right)^{2}}{\left(1-r^{2}\right)^{2}}\right\},
\end{aligned}
$$

and

$$
B_{3}:\left\{y:\left(y_{1}-\frac{x_{1}-2 \sqrt{3} r^{2}+x_{1} r^{2}}{1-r^{2}}\right)^{2}+\left(y_{2}-x_{2}\right)^{2}<\frac{4 r^{2}\left(x_{1}-\sqrt{3}\right)^{2}}{\left(1-r^{2}\right)^{2}}\right\} .
$$


Hence, $B_{s_{G}}(x, r)$ is smooth if and only if $B_{s_{G}}(x, r)$ is one of the above three balls. It is known that $\mathbf{B}^{2}\left(a, r_{1}\right) \subset \mathbf{B}^{2}\left(b, r_{2}\right)$ is equivalent to $|a-b| \leq r_{2}-r_{1}$. Then, by calculations, we have that for any point $x=\left(x_{1}, x_{2}\right) \in G, B_{1} \subset B_{2}$ and $B_{1} \subset B_{3}$ is equivalent to

$$
0<r \leq \frac{2 x_{2}}{\sqrt{x_{1}^{2}+x_{2}^{2}}}, \quad \text { and } \quad 0<r \leq \frac{x_{2}-\sqrt{3} x_{1}+2}{\sqrt{\left(\sqrt{3}-x_{1}\right)^{2}+\left(1-x_{2}\right)^{2}}}
$$

$B_{2} \subset B_{1}$ and $B_{2} \subset B_{3}$ is equivalent to

$$
0<r \leq-\frac{2 x_{2}}{\sqrt{x_{1}^{2}+x_{2}^{2}}}, \quad \text { and } \quad 0<r \leq \frac{-x_{2}-\sqrt{3} x_{1}+2}{\sqrt{\left(\sqrt{3}-x_{1}\right)^{2}+\left(1+x_{2}\right)^{2}}} ;
$$

$B_{3} \subset B_{1}$ and $B_{3} \subset B_{2}$ is equivalent to

$$
0<r \leq \frac{\sqrt{3} x_{1}-x_{2}-2}{\sqrt{\left(\sqrt{3}-x_{1}\right)^{2}+\left(1-x_{2}\right)^{2}}}, \quad \text { and } \quad 0<r \leq \frac{\sqrt{3} x_{1}+x_{2}-2}{\sqrt{\left(\sqrt{3}-x_{1}\right)^{2}+\left(1+x_{2}\right)^{2}}} .
$$

That is for any point $x \in T_{\frac{\pi}{6}, 2}, 0<r<1, B_{s_{G}}(x, r)$ is smooth if and only if

$$
0<r \leq \min \left\{\frac{2\left|x_{2}\right|}{\sqrt{x_{1}^{2}+x_{2}^{2}}}, \frac{\left|x_{2}\right|-\sqrt{3} x_{1}+2}{\sqrt{\left(x_{1}-\sqrt{3}\right)^{2}+\left(1-\left|x_{2}\right|\right)^{2}}}\right\}
$$

or

$$
0<r \leq \frac{\sqrt{3} x_{1}-2-\left|x_{2}\right|}{\sqrt{\left(x_{1}-\sqrt{3}\right)^{2}+\left(1-\left|x_{2}\right|\right)^{2}}}
$$

Obviously, for $x_{2}=0$ and $0<x_{1} \leq \frac{2 \sqrt{3}}{3}$, or $\left|x_{2}\right|=\sqrt{3} x_{1}-2, B_{s_{G}}(x, r)$ cannot be smooth.

For the case $G=R_{a, b}$, let $l_{1}: y_{2}=b, l_{2}: y_{1}=a, l_{3}: y_{2}=-b$, and $l_{4}: y_{1}=-a$. For any point $x \in R_{a, b}$, and $r \in(0,1)$, it follows from Lemma 4.1 that

$$
B_{s_{G}}(x, r)=\bigcap_{i=1}^{4} B_{i},
$$

where $B_{i}$ is the corresponding triangular ratio metric ball $B_{s_{G_{i}}}(x, r)$, and $G_{i}$ is the half plane with boundary line $l_{i}$. For any point $x \in R_{a, b}$, it follows from elementary computation that

$$
\begin{aligned}
& B_{1}:\left\{y:\left(y_{1}-x_{1}\right)^{2}+\left(y_{2}-\frac{x_{2}+r^{2} x_{2}-2 b r^{2}}{1-r^{2}}\right)^{2}<\frac{4 r^{2}\left(b-x_{2}\right)^{2}}{\left(1-r^{2}\right)^{2}}\right\}, \\
& B_{2}:\left\{y:\left(y_{1}-\frac{x_{1}+r^{2} x_{1}-2 a r^{2}}{1-r^{2}}\right)^{2}+\left(y_{2}-x_{2}\right)^{2}<\frac{4 r^{2}\left(a-x_{1}\right)^{2}}{\left(1-r^{2}\right)^{2}}\right\}, \\
& B_{3}:\left\{y:\left(y_{1}-x_{1}\right)^{2}+\left(y_{2}-\frac{x_{2}+r^{2} x_{2}+2 b r^{2}}{1-r^{2}}\right)^{2}<\frac{4 r^{2}\left(b+x_{2}\right)^{2}}{\left(1-r^{2}\right)^{2}}\right\},
\end{aligned}
$$


and

$$
B_{4}:\left\{y:\left(y_{1}-\frac{x_{1}+r^{2} x_{1}+2 a r^{2}}{1-r^{2}}\right)^{2}+\left(y_{2}-x_{2}\right)^{2}<\frac{4 r^{2}\left(a+x_{1}\right)^{2}}{\left(1-r^{2}\right)^{2}}\right\} .
$$

For $1 \leq i \leq 4$, let $R_{i}$ denote the radius of $B_{i}$. If $x_{2}>0$, then $R_{3} \geq R_{1}$. By calculations, $B_{s_{G}}(x, r)=B_{1}$ is equivalent to

$$
0<r \leq \min \left\{\frac{x_{2}}{b}, \frac{\left(a-x_{1}\right)-\left(b-x_{2}\right)}{\sqrt{\left(a-x_{1}\right)^{2}+\left(b-x_{2}\right)^{2}}}, \frac{\left(a+x_{1}\right)-\left(b-x_{2}\right)}{\sqrt{\left(a+x_{1}\right)^{2}+\left(b-x_{2}\right)^{2}}}\right\} .
$$

If $x_{2}<0$, then $R_{1} \geq R_{3}$. By calculations, $B_{s_{G}}(x, r)=B_{3}$ is equivalent to

$$
0<r \leq \min \left\{-\frac{x_{2}}{b}, \frac{\left(a-x_{1}\right)-\left(b+x_{2}\right)}{\sqrt{\left(a-x_{1}\right)^{2}+\left(b+x_{2}\right)^{2}}}, \frac{\left(a+x_{1}\right)-\left(b+x_{2}\right)}{\sqrt{\left(a+x_{1}\right)^{2}+\left(b+x_{2}\right)^{2}}}\right\} .
$$

If $x_{1}>0$, then $R_{4} \geq R_{2}$. By calculations, $B_{s_{G}}(x, r)=B_{2}$ is equivalent to

$$
0<r \leq \min \left\{\frac{x_{1}}{a}, \frac{\left(b-x_{2}\right)-\left(a-x_{1}\right)}{\sqrt{\left(a-x_{1}\right)^{2}+\left(b-x_{2}\right)^{2}}}, \frac{\left(b+x_{2}\right)-\left(a-x_{1}\right)}{\sqrt{\left(a-x_{1}\right)^{2}+\left(b+x_{2}\right)^{2}}}\right\} .
$$

If $x_{1}<0$, then $R_{2} \geq R_{4}$. By calculations, $B_{s_{G}}(x, r)=B_{4}$ is equivalent to

$$
0<r \leq \min \left\{-\frac{x_{1}}{a}, \frac{\left(b-x_{2}\right)-\left(a+x_{1}\right)}{\sqrt{\left(a+x_{1}\right)^{2}+\left(b-x_{2}\right)^{2}}}, \frac{\left(b+x_{2}\right)-\left(a+x_{1}\right)}{\sqrt{\left(a+x_{1}\right)^{2}+\left(b+x_{2}\right)^{2}}}\right\} .
$$

That is, for any point $x \in R_{a, b}, 0<r<1, B_{s_{G}}(x, r)$ is smooth if and only if

$$
0<r \leq \min \left\{\frac{\left|x_{2}\right|}{b}, \frac{\left(a-\left|x_{1}\right|\right)-\left(b-\left|x_{2}\right|\right)}{\sqrt{\left(a-\left|x_{1}\right|\right)^{2}+\left(b-\left|x_{2}\right|\right)^{2}}}\right\} \text {, }
$$

or

$$
0<r \leq \min \left\{\frac{\left|x_{1}\right|}{a}, \frac{\left(b-\left|x_{2}\right|\right)-\left(a-\left|x_{1}\right|\right)}{\sqrt{\left(a-\left|x_{1}\right|\right)^{2}+\left(b-\left|x_{2}\right|\right)^{2}}}\right\} .
$$

Obviously, for $x_{2}=0$ and $a-\left|x_{1}\right| \geq b$, or $a-\left|x_{1}\right|=b-\left|x_{2}\right|, B_{s_{G}}(x, r)$ cannot be smooth.

\section{Quasiregular maps and triangular ratio metric}

In this section our goal is to summarize some basic facts about quasiconformal mappings, following closely [AVV], and [Vu1], and to prove Theorems 1.2 and 1.3. We assume that the reader is familiar with the basics of this theory. Here we adopt the standard definition of $K$-quasiconformality and $K$-quasiregularity from Väisälä's book [V] and from [Vu1], respectively. The first result is a quasiregular counterpart of the Schwarz lemma. Observe that the result is asymptotically sharp when $K \rightarrow 1$.

The Grötzsch ring domain $R_{G, n}(s), s>1$, is a doubly connected domain with complementary components $\left(\overline{\mathbf{B}^{n}},\left[s e_{1}, \infty\right)\right)$. For its capacity we write

$$
\gamma_{n}(s)=\operatorname{cap} R_{G, n}(s)=M\left(\Delta\left(\overline{\mathbf{B}^{n}},\left[s e_{1}, \infty\right]\right)\right)
$$


For $K>0$ we define an increasing homeomorphism $\varphi_{K, n}:[0,1] \rightarrow[0,1]$ with $\varphi_{K, n}(0)=0, \varphi_{K, n}(1)=1$ and

$$
\varphi_{K, n}(r)=\frac{1}{\gamma_{n}^{-1}\left(K \gamma_{n}(1 / r)\right)}, \quad 0<r<1
$$

The following important estimates are well known [Vu1, pp. 98-99]

$$
\begin{aligned}
r^{\alpha} \leq \varphi_{K, n}(r) & \leq \lambda_{n}^{1-\alpha} r^{\alpha} \leq 2^{1-1 / K} K r^{\alpha}, \quad \alpha=K^{1 /(1-n)}, \\
2^{1-K} K^{-K} r^{\beta} & \leq \lambda_{n}^{1-\beta} r^{\beta} \leq \varphi_{1 / K, n}(r) \leq r^{\beta}, \quad \beta=1 / \alpha,
\end{aligned}
$$

where $K \geq 1, r \in(0,1)$, and the constant $\lambda_{n} \in\left[4,2 e^{n-1}\right)$ is the so-called Grötzsch ring constant. In particular, $\lambda_{2}=4$.

Theorem 5.4. Let $G, D$ be either $\mathbf{B}^{n}$ or $\mathbf{H}^{n}$ and $f: G \rightarrow f G \subset D$ be a nonconstant $K$-quasiregular mapping and let $\alpha=K_{I}(f)^{1 /(1-n)}$. Then

$$
\operatorname{th}\left(\frac{1}{2} \rho_{D}(f(x), f(y))\right) \leq \varphi_{K, n}\left(\operatorname{th}\left(\frac{1}{2} \rho_{G}(x, y)\right)\right) \leq \lambda_{n}^{1-\alpha}\left(\operatorname{th}\left(\frac{1}{2} \rho_{G}(x, y)\right)\right)^{\alpha}
$$

for all $x, y \in G$.

Proof. Recall that the proof in [Vu1, Theorem 11.2] for the case $G=D=\mathbf{B}^{n}$ was based on the formula

$$
\mu_{\mathbf{B}^{n}}(x, y)=\gamma_{n}\left(\frac{1}{\operatorname{th} \frac{\rho_{\mathbf{B}^{n}(x, y)}}{2}}\right), \quad x, y \in \mathbf{B}^{n} .
$$

and the transformation rule of the metric $\mu_{\mathbf{B}^{n}}$ under quasiregular maps. The same proof also works for the present general case as soon as we prove that the formula (5.5) also holds for the case of $\mathbf{H}^{n}$. For this purpose we use the invariance of $\mu_{\mathbf{B}^{n}}$ under a Möbius transformation $h: \mathbf{H}^{n} \rightarrow \mathbf{B}^{n}$ to conclude by (5.5) that for $x, y \in \mathbf{H}^{n}$,

$$
\mu_{\mathbf{H}^{n}}(x, y)=\mu_{\mathbf{B}^{n}}(h(x), h(y))=\gamma_{n}\left(\frac{1}{\operatorname{th} \frac{\rho_{\mathbf{B}^{n}(h(x), h(y))}}{2}}\right)=\gamma_{n}\left(\frac{1}{\operatorname{th} \frac{\rho_{\mathbf{H}^{n}(x, y)}}{2}}\right),
$$

where in the last step we used the invariance of the hyperbolic metric under the Möbius transformation $h$, see [Vu1, (2.21)]. After these observations the proof goes in the same way as in [Vu1, Theorem 11.2].

Proof of Theorem 1.2. (1) Because for all $x, y \in \mathbf{H}^{n}$,

$$
s_{\mathbf{H}^{n}}(x, y)=\operatorname{th}\left(\frac{\rho_{\mathbf{H}^{n}}(x, y)}{2}\right),
$$

by Theorem 5.4 the proof follows.

(2) By Theorems 5.4, 3.23 and Lemma 3.8 we have for all $x, y \in \mathbf{B}^{n}$,

$$
\begin{aligned}
s_{\mathbf{B}^{n}}(f(x), f(y)) & \leq \operatorname{th}\left(\frac{\rho_{\mathbf{B}^{n}}(f(x), f(y))}{2}\right) \leq \lambda_{n}^{1-\alpha} \operatorname{th}\left(\frac{\rho_{\mathbf{B}^{n}}(x, y)}{2}\right)^{\alpha} \\
& \leq \lambda_{n}^{1-\alpha}\left(2 s_{\mathbf{B}^{n}}(x, y)\right)^{\alpha}=2^{\alpha} \lambda_{n}^{1-\alpha}\left(s_{\mathbf{B}^{n}}(x, y)\right)^{\alpha} .
\end{aligned}
$$


(3) Similarly by Theorems 5.4 and 3.23 we have for all $x, y \in \mathbf{B}^{n}$,

$$
\begin{aligned}
s_{\mathbf{H}^{n}}(f(x), f(y)) & =\operatorname{th}\left(\frac{\rho_{\mathbf{H}^{n}}(f(x), f(y))}{2}\right) \leq \lambda_{n}^{1-\alpha} \operatorname{th}\left(\frac{\rho_{\mathbf{B}^{n}}(x, y)}{2}\right)^{\alpha} \\
& \leq \lambda_{n}^{1-\alpha}\left(2 s_{\mathbf{B}^{n}}(x, y)\right)^{\alpha}=2^{\alpha} \lambda_{n}^{1-\alpha}\left(s_{\mathbf{B}^{n}}(x, y)\right)^{\alpha} .
\end{aligned}
$$

(4) By Theorems 3.4, 3.8 and 5.4 we have for all $x, y \in \mathbf{H}^{n}$,

$$
\begin{aligned}
s_{\mathbf{B}^{n}}(f(x), f(y)) & \leq \operatorname{th}\left(\frac{\rho_{\mathbf{B}^{n}}(f(x), f(y))}{2}\right) \\
& \leq \lambda_{n}^{1-\alpha} \operatorname{th}\left(\frac{\rho_{\mathbf{H}^{n}}(x, y)}{2}\right)^{\alpha}=\lambda_{n}^{1-\alpha}\left(s_{\mathbf{H}^{n}}(x, y)\right)^{\alpha} .
\end{aligned}
$$

Theorem 5.6. Let $f: \mathbf{B}^{n} \rightarrow \mathbf{B}^{n}$ be a $K$-quasiregular mapping. Then for $x, y \in$ $\mathbf{B}^{n}$ we have

$$
p_{\mathbf{B}^{n}}(f(x), f(y)) \leq 2^{\alpha} \lambda_{n}^{1-\alpha}\left(p_{\mathbf{B}^{n}}(x, y)\right)^{\alpha}, \quad \alpha=K^{1 /(1-n)} .
$$

Proof. By Lemma 3.8, the proof is similar to the proof of Theorem 1.2.

By definition (1.1) it is clear that for $x, y \in G=\mathbf{R}^{n} \backslash\{0\}$, we have

$$
s_{G}(x, y)=\frac{|x-y|}{|x|+|y|} .
$$

Recall the following notation from [AVV, Section 14],

$$
\begin{gathered}
\eta_{K, n}^{*}(t)=\sup \left\{|g(x)|:|x| \leq t, g \in \mathcal{F}_{K}\right\}, \\
\mathcal{F}_{K}=\left\{g: \mathbf{R}^{n} \rightarrow \mathbf{R}^{n}, g(0)=0, g\left(e_{1}\right)=e_{1}, g \text { is } K \text {-quasiconformal }\right\} .
\end{gathered}
$$

Lemma 5.8. [AVV, 14.27] Let $f: \mathbf{R}^{n} \rightarrow \mathbf{R}^{n}$ be a $K$-quasiconformal mapping with $f(\infty)=\infty$, and let $a, b, c$ be three distinct points in $\mathbf{R}^{n}$. Then

$$
\begin{aligned}
\frac{1}{P_{6}(n, K)}\left(\frac{|a-c|}{|a-b|+|b-c|}\right)^{\beta} & \leq \frac{|f(a)-f(c)|}{|f(a)-f(b)|+|f(b)-f(c)|} \\
& \leq \frac{1}{P_{5}(n, K)}\left(\frac{|a-c|}{|a-b|+|b-c|}\right)^{\alpha}
\end{aligned}
$$

where $\alpha=K^{1 /(1-n)}=1 / \beta$ and $P_{5}(n, K)=2^{1-(\beta / \alpha)} \lambda_{n}^{1-\beta} / \eta_{K, n}^{*}(1)$ and $P_{6}(n, K)=$ $2^{1-(\alpha / \beta)} \lambda_{n}^{\beta-1} \eta_{K, n}^{*}(1)$. Here $\lambda_{n}$ is as in Lemma 5.4 and $P_{5}(n, K) \rightarrow 1, P_{6}(n, K) \rightarrow 1$, when $K \rightarrow 1$.

Proof of Theorem 1.3. By Möbius invariance of the absolute ratio, the result follows from Lemma 5.8 if we take $b=f(b)=0$.

Lemma 5.9. [AVV, 14.8] For $n \geq 2$ and $K \geq 1$,

$$
\eta_{K, n}^{*}(1) \leq \exp (4 K(K+1) \sqrt{K-1}) .
$$

Corollary 5.10. Let $G=\mathbf{R}^{n} \backslash\{0\}$, and $f: G \rightarrow G$ be a $K$-quasiconformal mapping. If $n \geq 2, \alpha=K^{1 /(1-n)}$, then for $z, w \in G$,

$$
s_{f G}(f(z), f(w)) \leq K^{K} \exp (2(K+1)(K-1)+4 K(K+1) \sqrt{K-1})\left(s_{G}(z, w)\right)^{\alpha} .
$$


Proof. Combining Lemmas 5.8 and 5.9 and by [Vu1, Lemma 7.50 (2)] we see that

$$
\begin{aligned}
\frac{1}{P_{5}(n, K)} & =\frac{\eta_{K, n}^{*}(1)}{2^{1-(\beta / \alpha)} \lambda_{n}^{1-\beta}} \leq \frac{2^{K-1} K^{K} \eta_{K, n}^{*}(1)}{2^{1-(\beta / \alpha)}} \leq 2^{(\beta / \alpha)+K-2} K^{K} \eta_{K, n}^{*}(1) \\
& \leq 2^{(\beta / \alpha)+K-2} K^{K} \exp (4 K(K+1) \sqrt{K-1}) \\
& \leq K^{K} \exp ((\beta / \alpha)+K-2+4 K(K+1) \sqrt{K-1}) \\
& \leq K^{K} \exp (2(K+1)(K-1)+4 K(K+1) \sqrt{K-1}) .
\end{aligned}
$$

Acknowledgements. The authors are indebted to the referee for a very valuable set of corrections. This research was supported by the Academy of Finland, Project 2600066611 and the Väisälä foundation. The research of the first author was supported by CIMO and China Scholarship Council. The research of the second author was supported also by CIMO. The research of the third author was supported also by the Marsden Fund, New Zealand.

\section{References}

[AVV] Anderson, G. D., M. K. Vamanamurthy, and M. Vuorinen: Conformal invariants, inequalities and quasiconformal maps. - J. Wiley, 1997.

[BA] BArrlund, A.: The p-relative distance is a metric. - SIAM J. Matrix Anal. Appl. 21:2, 1999, 699-702.

[B] Beardon, A.F.: The geometry of discrete groups. - Graduate Texts in Math. 91, Springer-Verlag, New York, 1983.

[DC] Deng, J.-E., and C.-P. Chen: Sharp Shafer-Fink type inequalities for Gauss lemniscate functions. - J. Inequal. Appl. 2014:35, 2014.

[H] HästÖ, P.: A new weighted metric, the relative metric I. - J. Math. Anal. Appl. 274, 2002, 38-58.

[HIMPS] Hästö, P., Z. Ibragimov, D. Minda, S. Ponnusamy, and S. K. Sahoo: Isometries of some hyperbolic-type path metrics, and the hyperbolic medial axis. - In: In the tradition of Ahlfors-Bers IV, Contemp. Math. 432, 2007, 63-74.

[HKLV] HoKuni, S., R. KLÉN, Y. LI, and M. VuORINEn: Balls in the triangular ratio metric. In: Proceedings of the International conference Complex Analysis and Dynamical Systems VI, Contemp. Math. (to appear).

[KL] KeEn, L., and N. LAKIC: Hyperbolic geometry from a local viewpoint. - London Math. Soc. Student Texts 68, Cambridge Univ. Press, Cambridge, 2007.

[KLVW] KlÉn, R., H. Lindén, M. Vuorinen, and G. WAng: The visual angle metric and Möbius transformations. - Comput. Methods Funct. Theory 14, 2014, 577-608.

[PT] Papadopoulos, A., and M. Troyanov: Weak metrics on Euclidean domains. - JP J. Geom. Topol. 7:1, 2007, 23-44.

[V] VÄısÄLÄ, J.: Lectures on n-dimensional quasiconformal mappings. - Lecture Notes in Math. 229, Springer-Verlag, Berlin-Heidelberg-New York, 1971.

[Vu1] Vuorinen, M.: Conformal geometry and quasiregular mappings. - Lecture Notes in Math. 1319, Springer-Verlag, Berlin, 1988.

[Vu2] Vuorinen, M.: Geometry of metrics. - In: Proc. ICM2010 Satellite Conf. International Workshop on Harmonic and Quasiconformal Mappings (HMQ2010), eds. D. Minda, S. Ponnusamy, N. Shanmugalingam, J. Anal. 18, 2010, 399-424.

Received 27 March 2014 • First revised received 7 September 2014

Second revised received 5 January $2015 \bullet$ Accepted 12 January 2015 